\title{
Unveiling the nature of INTEGRAL objects through optical spectroscopy ${ }^{\star}$
}

\section{Identification of 20 Galactic and extragalactic hard X-ray sources}

\author{
N. Masetti ${ }^{1}$, P. Parisi ${ }^{1,2}$, E. Palazzi ${ }^{1}$, E. Jiménez-Bailón ${ }^{3}$, L. Morelli ${ }^{4}$, V. Chavushyan ${ }^{5}$, E. Mason ${ }^{6}$, V. A. McBride ${ }^{7}$, \\ L. Bassani ${ }^{1}$, A. Bazzano ${ }^{8}$, A. J. Bird ${ }^{7}$, A. J. Dean ${ }^{7}$, G. Galaz ${ }^{9}$, N. Gehrels ${ }^{10}$, R. Landi ${ }^{1}$, A. Malizia ${ }^{1}$, D. Minniti ${ }^{9}, 11$, \\ F. Schiavone ${ }^{1}$, J. B. Stephen ${ }^{1}$, and P. Ubertini ${ }^{8}$
}

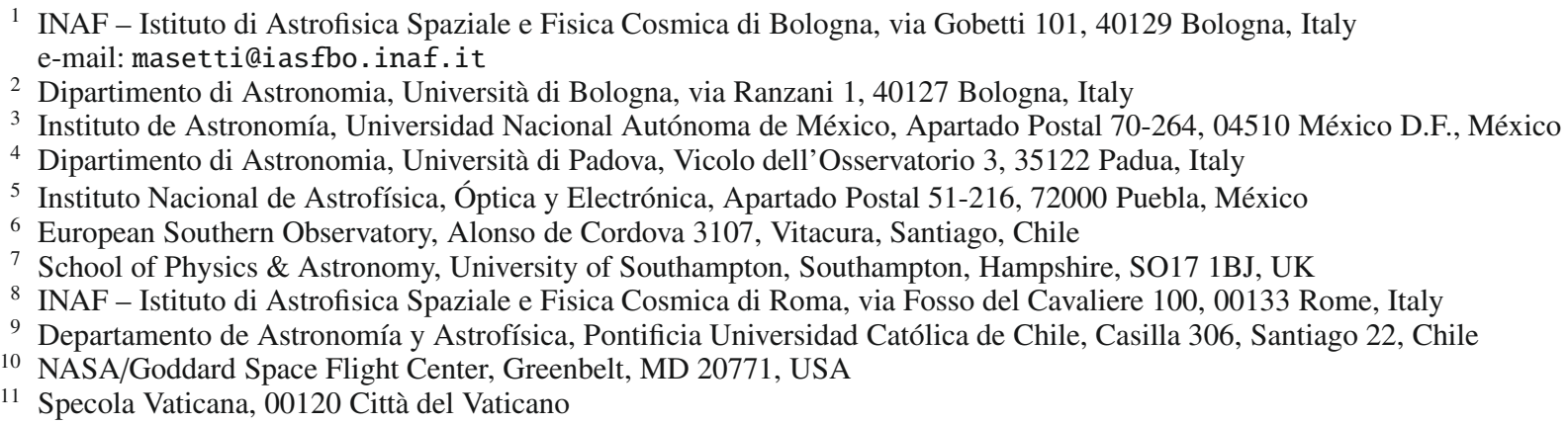

Received 10 November 2008/ Accepted 19 November 2008

\section{ABSTRACT}

\begin{abstract}
Within the framework of our program of assessment of the nature of unidentified or poorly known INTEGRAL sources, we present here spectroscopy of optical objects, selected through positional cross-correlation with soft X-ray detections (afforded with satellites such as Swift, ROSAT, Chandra and/or XMM-Newton) as putative counterparts of hard X-ray sources detected with the IBIS instrument onboard INTEGRAL. Using 6 telescopes of various sizes and archival data from two on-line spectroscopic surveys we are able to identify, either for the first time or independent of other groups, the nature of 20 INTEGRAL hard X-ray sources. Our results indicate that: 11 of these objects are active galactic nuclei (AGNs) at redshifts between 0.014 and $0.978,7$ of which display broad emission lines, 2 show narrow emission lines only, and 2 have unremarkable or no emission lines (thus are likely Compton thick AGNs); 5 are cataclysmic variables (CVs), 4 of which are (possibly magnetic) dwarf novae and one is a symbiotic star; and 4 are Galactic $\mathrm{X}$-ray binaries ( 3 with high-mass companions and one with a low-mass secondary). It is thus again found that the majority of these sources are AGNs or magnetic CVs, confirming our previous findings. When possible, the main physical parameters for these hard $\mathrm{X}$-ray sources are also computed using the multiwavelength information available in the literature. These identifications support the importance of INTEGRAL in the study of the hard X-ray spectrum of all classes of X-ray emitting objects, and the effectiveness of a strategy of multi-catalogue cross-correlation plus optical spectroscopy to securely pinpoint the actual nature of unidentified hard $\mathrm{X}$-ray sources.
\end{abstract}

Key words. galaxies: Seyfert - quasars: emission lines - X-rays: binaries - stars: novae, cataclysmic variables techniques: spectroscopic - X-rays: general

\section{Introduction}

One of the fundamental aims of the INTEGRAL mission (Winkler et al. 2003) is the census of the whole sky in the hard $\mathrm{X}$-ray band above $20 \mathrm{keV}$. This makes use of the unique imaging capability of the IBIS instrument (Ubertini et al. 2003) which

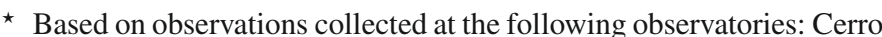
Tololo Interamerican Observatory (Chile); Observatorio del Roque de los Muchachos of the Instituto de Astrofísica de Canarias (Canary Islands, Spain); ESO (La Silla, Chile) under programme 079.A0171(A); Astronomical Observatory of Bologna in Loiano (Italy); Observatorio Astronómico Nacional (San Pedro Mártir, México); Anglo-Australian Observatory (Siding Spring, Australia); Apache Point Observatory (New Mexico, USA).
}

allows the detection of sources at the mCrab level with a typical localization accuracy of less than 5 arcmin (Gros et al. 2003).

The IBIS surveys secured the detection of extragalactic sources in the so-called "Zone of Avoidance", which hampers observations in soft X-rays along the Galactic Plane due to the presence of gas and dust. Moreover, these surveys are expanding our knowledge about Galactic X-ray binaries, by showing the existence of a new class of heavily absorbed supergiant massive X-ray binaries (first suggested by Revnivtsev et al. 2003), by allowing the discovery and the study of supergiant fast X-ray transients (e.g., Sguera et al. 2006; Leyder et al. 2007; Sidoli et al. 2008), by doubling the number of known high-mass X-ray binaries (HMXBs, see Walter 2007), and by detecting a substantial number of new magnetic cataclysmic variables $(\mathrm{CVs}$, 
e.g., Barlow et al. 2006; Bonnet-Bidaud et al. 2007; Landi et al. 2008a).

Up to now, IBIS has detected more than 500 sources at hard X-rays between 20 and $100 \mathrm{keV}$ (see e.g., Bird et al. 2007; Krivonos et al. 2007; see also Bodaghee et al. 2007). If we consider the most complete compilation of IBIS sources available (Bodaghee et al. 2007; see also Bird et al. 2007), one can see that most of these sources are active galactic nuclei (AGNs, $33 \%$ of the total number of detected objects) followed by known Galactic X-ray binaries (32\%) and CVs (4.5\%). However, a large number of the remaining objects ( $26 \%$ of all IBIS detections, according to Bodaghee et al. 2007) has no obvious counterpart at other wavelengths and therefore cannot immediately be associated with any known class of high-energy emitting objects. The multiwavelength study of these unidentified sources is thus critical to determine their nature.

Therefore, in 2004 we started a multisite observational campaign devoted to the identification of these unidentified objects through optical spectroscopy (see Masetti et al. 2004, 2006a,b,c,d, 2008a, hereafter Papers I-VI). Our results showed that about half of these objects are nearby $(z \lesssim 0.1)$ AGNs (Papers I-VI), while a non-negligible fraction $(\sim 15 \%)$ of objects belongs to the class of magnetic CVs (Paper VI and references therein). The results of this identification program, along with those obtained by other groups, are collected as a service to the scientific community on a web page ${ }^{1}$ reporting information on INTEGRAL sources identified via optical or near-infrared (NIR) observations.

Continuing our effort to reveal the real nature of INTEGRAL sources, we present here optical spectroscopy of 20 objects that were detected by IBIS but are still unidentified, unclassified or poorly studied. The data reported here were obtained at 6 different telescopes around the world, or retrieved from two public spectroscopic archives.

A thorough description of the approach to the sample selection is reported in Papers I-VI, so we refer the reader to these papers for details on the procedure and caveats thereof. Here we simply state that all of the optical counterparts to the IBIS sources studied in this paper are positionally associated with a soft X-ray object detected with ROSAT (Voges et al. 1999; ROSAT Team 2000), Swift/XRT ${ }^{2}$ (Malizia et al. 2007; Landi et al. 2007a,b, 2008b,c,d,e; Revnivtsev et al. 2007; Rodriguez et al. 2008), Chandra (Jonker \& Kuiper 2007; Sazonov et al. 2008; Tomsick et al. 2008a,b), and/or XMM-Newton (Saxton et al. 2008; Watson et al. 2008; Ibarra et al. 2008a,b) within the corresponding INTEGRAL error circle (Bird et al. 2007; Krivonos et al. 2007; Sazonov et al. 2008).

We remark that this approach is highly effective, as we have already sucessfully identified more than 80 selected targets (see Papers I-VI; Masetti et al. 2007, 2008b).

For the high-energy sources with more than one optical candidate, we spectroscopically observed all objects with magnitude $R \lesssim 18$ within the corresponding soft X-ray arcsec-sized error circle. However, in the following we will report only on their firm or likely optical counterparts, recognized via their peculiar spectral features (basically, the presence of emission lines). All other candidates were excluded because their spectra did not show any peculiarity (in general they are recognized as Galactic stars) and will not be considered further.

\footnotetext{
${ }^{1}$ http://www.iasfbo.inaf.it/extras/IGR/main.html

2 XRT archival data are freely available at

http://www. asdc.asi.it/
}

We note that we included in our list of objects the hard X-ray source IGR J12415-5750, which is reported as a Seyfert 2 AGN in Bird et al. (2007) but for which no optical spectrum is available in the literature (Winter et al. 2008). We also considered IGR J19267+1325, which has a soft X-ray source close to (but formally outside of) its $90 \%$ IBIS error circle; it can nevertheless be considered the likely soft X-ray counterpart of this INTEGRAL source on the basis of $\mathrm{X}$-ray spectral comparisons (Tomsick et al. 2008b).

In our final sample of sources we also included the INTEGRAL objects mentioned by Negueruela et al. (2007), Bikmaev et al. (2008a,b), Revnivtsev et al. (2008) and Steeghs et al. (2008). These objects, although already identified elsewhere, still have fragmentary longer-wavelength information, or were independently studied by us before their identification was announced. Our observations are thus meant to confirm their nature and to improve their classification and known information.

Figures 1 and 2 report the optical finding charts of the 20 sources of the selected sample. The corresponding optical counterparts are indicated with tick marks. The list of identified INTEGRAL sources is reported in Table 1 (which we thoroughly describe in the next section).

The outline of the rest of the paper is the following: in Sect. 2 a description of the observations and of the employed telescopes is given. Section 3 reports and discusses the results, divided into three broad classes of sources (CVs, X-ray binaries and AGNs), together with an update of the statistical outline of the identifications of INTEGRAL sources obtained until now. Conclusions are drawn in Sect. 4. In this work, if not otherwise stated, errors and limits are reported at $1 \sigma$ and $3 \sigma$ confidence levels, respectively.

\section{Optical spectroscopy}

As in the case of Paper VI, the data presented in this paper were collected in the course of a campaign that involved observations at the following telescopes in the past 2 years:

\section{- the $1.5 \mathrm{~m}$ at the Cerro Tololo Interamerican Observatory (CTIO), Chile;}

- the $1.52 \mathrm{~m}$ "Cassini" telescope of the Astronomical Observatory of Bologna, in Loiano, Italy;

- the $2.1 \mathrm{~m}$ telescope of the Observatorio Astronómico Nacional in San Pedro Martir, México;

- the 3.6 m telescope at the ESO-La Silla Observatory, Chile;

- the $3.58 \mathrm{~m}$ "Telescopio Nazionale Galileo" (TNG) and the $4.2 \mathrm{~m}$ "William Herschel Telescope" (WHT) at the Roque de Los Muchachos Observatory in La Palma, Spain.

The spectroscopic data secured at these telescopes were optimally extracted (Horne 1986) and reduced following standard procedures using $\mathrm{IRAF}^{3}$. Calibration frames (flat fields and bias) were taken on the day preceeding or following the observing night. The wavelength calibration was obtained using lamps acquired soon after each on-target spectroscopic acquisiton; uncertainty on this calibration was $\sim 0.5 \AA$ for all cases: this was checked using the positions of background night sky lines. Flux calibration was performed using catalogued spectrophotometric standards.

\footnotetext{
3 IRAF is the Image Reduction and Analysis Facility made available to the astronomical community by the National Optical Astronomy Observatories, which are operated by AURA, Inc., under contract with the U.S. National Science Foundation. It is available at http://iraf.noao.edu/
} 

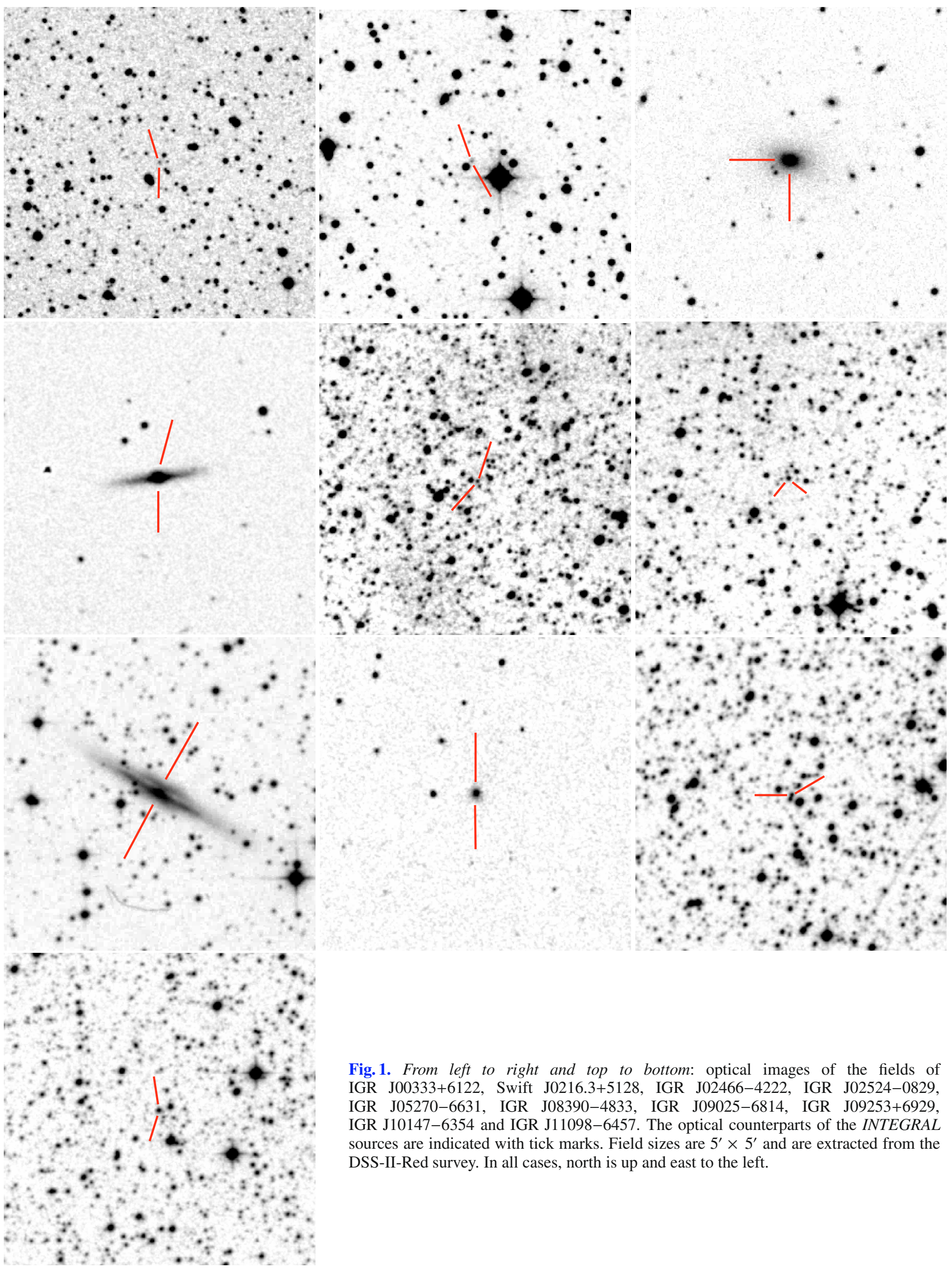

Fig. 1. From left to right and top to bottom: optical images of the fields of IGR J00333+6122, Swift J0216.3+5128, IGR J02466-4222, IGR J02524-0829, IGR J05270-6631, IGR J08390-4833, IGR J09025-6814, IGR J09253+6929, IGR J10147-6354 and IGR J11098-6457. The optical counterparts of the INTEGRAL sources are indicated with tick marks. Field sizes are $5^{\prime} \times 5^{\prime}$ and are extracted from the DSS-II-Red survey. In all cases, north is up and east to the left. 

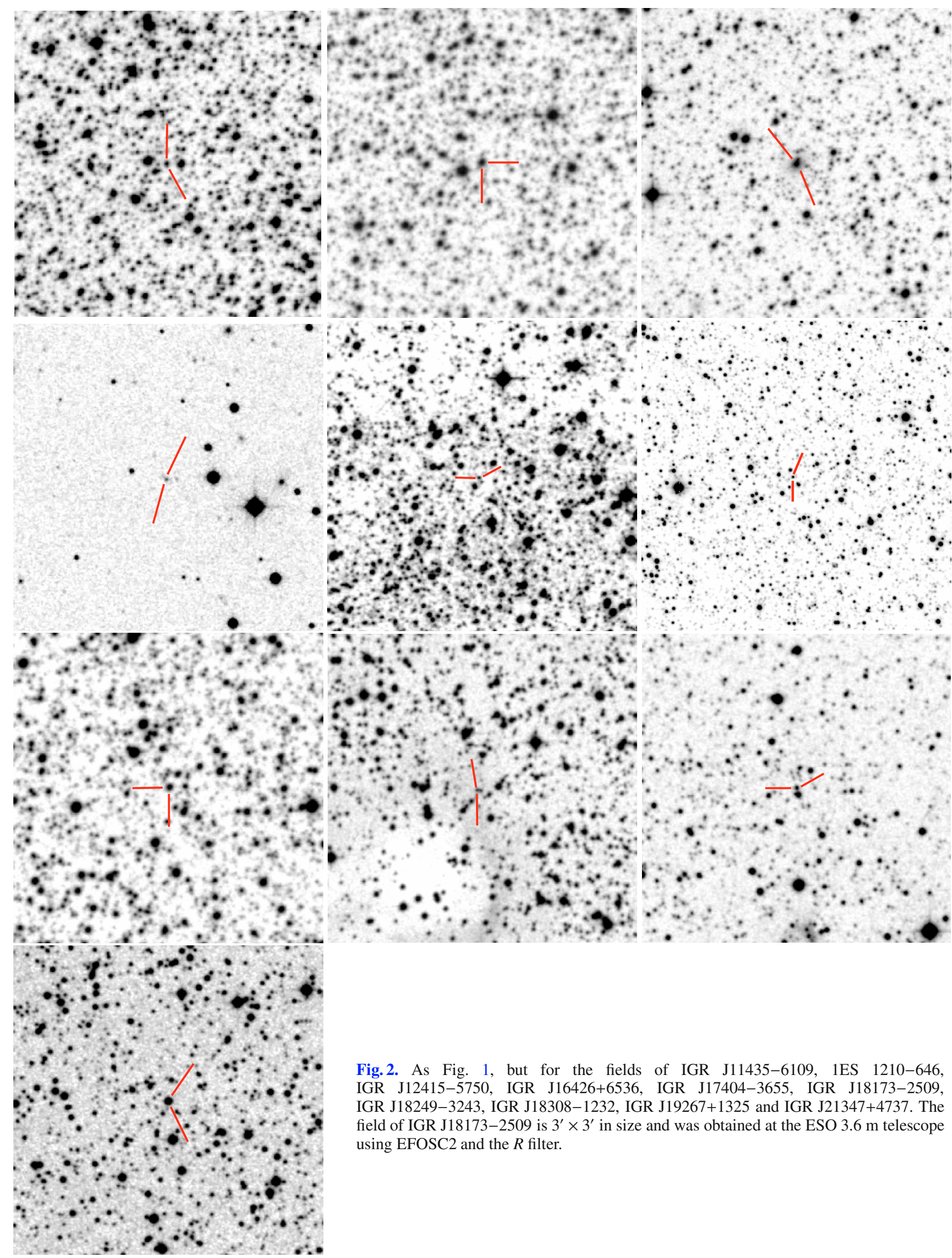
$6 x^{2}+0^{2}+0^{2}+0$
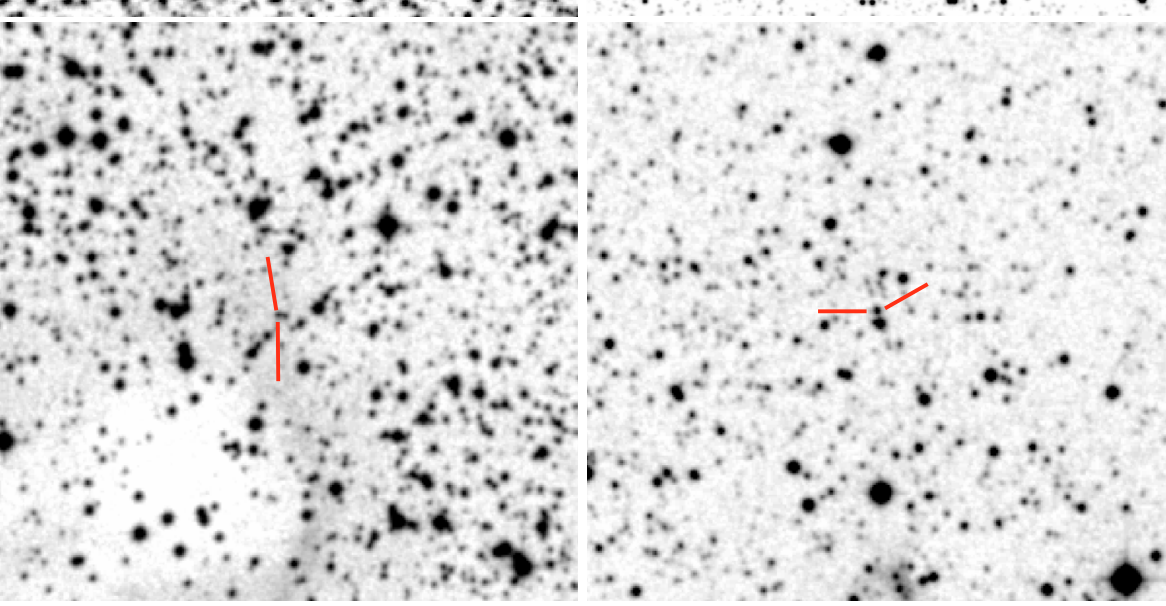

Fig. 2. As Fig. 1, but for the fields of IGR J11435-6109, 1ES 1210-646, IGR J12415-5750, IGR J16426+6536, IGR J17404-3655, IGR J18173-2509, IGR J18249-3243, IGR J18308-1232, IGR J19267+1325 and IGR J21347+4737. The field of IGR J18173-2509 is $3^{\prime} \times 3^{\prime}$ in size and was obtained at the ESO $3.6 \mathrm{~m}$ telescope using EFOSC 2 and the $R$ filter. 
Table 1. Log of the spectroscopic observations presented in this paper (see text for details). If not otherwise indicated, source coordinates are extracted from the 2MASS catalogue and have an accuracy better than $0 .{ }^{\prime} 1$.

\begin{tabular}{|c|c|c|c|c|c|c|c|}
\hline $\begin{array}{c}(1) \\
\text { Object }\end{array}$ & $\begin{array}{c}(2) \\
\text { RA } \\
(\mathrm{J} 2000)\end{array}$ & $\begin{array}{c}\text { (3) } \\
\text { Dec } \\
(\mathrm{J} 2000)\end{array}$ & $\begin{array}{c}\text { (4) } \\
\text { Telescope+instrument }\end{array}$ & $\begin{array}{c}(5) \\
\lambda \text { range } \\
(\AA)\end{array}$ & $\begin{array}{c}\text { (6) } \\
\text { Disp. } \\
(\AA / \text { pix })\end{array}$ & $\begin{array}{c}(7) \\
\text { UT date \& time } \\
\text { at mid-exposure }\end{array}$ & $\begin{array}{c}(8) \\
\text { Exposure } \\
\text { time (s) }\end{array}$ \\
\hline IGR J00333+6122 & $00: 33: 18.34$ & $+61: 27: 43.3$ & WHT+ISIS & $5050-10300$ & 1.8 & 05 Jan. 2008, 22:51 & $2 \times 1000$ \\
\hline Swift J0216.3+5128 & $02: 16: 26.73^{\dagger}$ & $+51: 25: 25.1^{\dagger}$ & WHT+ISIS & $5050-10300$ & 1.8 & 06 Jan. 2008, 00:30 & $2 \times 1800$ \\
\hline IGR J02466-4222 & $02: 46: 37.03$ & $-42: 22: 01.5$ & $\mathrm{AAT}+6 \mathrm{dF}$ & $3900-7600$ & 1.6 & 30 Aug. 2003, 18:06 & $1200+600$ \\
\hline IGR J02524-0829 & $02: 52: 23.39$ & $-08: 30: 37.5$ & SDSS+CCD Spc. & $3800-9200$ & 1.0 & 23 Dec. $2000,04: 07$ & 2700 \\
\hline IGR J05270-6631 & $05: 26: 14.45^{\dagger}$ & $-66: 30: 45.0^{\dagger}$ & 3.6 m+EFOSC & $3685-9315$ & 2.8 & 02 Jan. 2008, 06:19 & 900 \\
\hline IGR J08390-4833 & 08:38:49.11 & $-48: 31: 24.8$ & CTIO 1.5 m+RC Spec. & $3300-10500$ & 5.7 & 04 Mar. 2008, 05:19 & $2 \times 1800$ \\
\hline IGR J09025-6814 & 09:02:39.46 & $-68: 13: 36.6$ & CTIO 1.5 m+RC Spec. & $3300-10500$ & 5.7 & 19 Feb. 2007, 06:11 & $2 \times 1800$ \\
\hline IGR J09253+6929 & $09: 25: 47.56$ & $+69: 27: 53.6$ & Cassini+BFOSC & $3500-8700$ & 4.0 & 29 Mar. 2008, 21:13 & $2 \times 1800$ \\
\hline IGR J10147-6354 & $10: 14: 15.55$ & $-63: 51: 50.1$ & EFOSC & $3685-9315$ & 2.8 & 02 Jan. 2008, 06:44 & 900 \\
\hline IGR J11098-6457 & 11:09:47.79 & $-64: 52: 45.3$ & CTIO 1.5 m+RC Spec. & $3300-10500$ & 5.7 & 04 Mar. 2008, 06:44 & $2 \times 1200$ \\
\hline IGR J11435-6109 & $11: 44: 00.30$ & $-61: 07: 36.5$ & CTIO $1.5 \mathrm{~m}+\mathrm{RC}$ & $3300-10500$ & 5.7 & 01 Jul. 2008 & $2 \times 1200$ \\
\hline 1ES 1210-646 & $12: 13: 14.79$ & $-64: 52: 30.5$ & CTIO 1.5 m+RC Spec. & $3300-10500$ & 5.7 & 04 Mar. 2008 & $2 \times 900$ \\
\hline IGR J12415-5750 & $12: 41: 25.74$ & $-57: 50: 03.5$ & CTIO 1.5 m+RC Spec. & $3300-10500$ & 5.7 & 04 Mar. 2008 & $2 \times 1200$ \\
\hline IGR J16426+6536 & $16: 43: 04.07 \dagger$ & $+65: 32: 50.9 \dagger$ & TNG+DOLoRes & $3800-8000$ & 2.5 & 04 Feb. 2008, 06:11 & $2 \times 900$ \\
\hline IGR J17404-3655 & $17: 40: 26.86$ & $-36: 55: 37.4$ & CTIO 1.5 m+RC Spec. & $3300-10500$ & 5.7 & 02 Jul. 2008, 05:36 & $2 \times 2400$ \\
\hline IGR J18173-2509 & $18: 17: 22.3^{*}$ & $-25: 08: 43^{*}$ & CTIO 1.5 m+RC Spec. & $3300-10500$ & 5.7 & 02 Jul. 2008, 04:04 & $2 \times 2400$ \\
\hline IGR J18249-3243 & $18: 24: 55.92$ & $-32: 42: 57.7$ & CTIO 1.5 m+RC Spec. & $3300-10500$ & 5.7 & 04 Mar. 2008, 09:21 & $2 \times 1200$ \\
\hline IGR J18308-1232 & $18: 30: 49.88 \dagger$ & $-12: 32: 18.7 \dagger$ & SPM $2.1 \mathrm{~m}+\mathrm{B} \& \mathrm{C}$ Spec. & $3450-7650$ & 4.0 & 29 Jun. 2008, 07:49 & $3 \times 2400$ \\
\hline IGR J19267+1325 & $19: 26: 27.00$ & $+13: 22: 05.0$ & SPM $2.1 \mathrm{~m}+\mathrm{B} \& \mathrm{C}$ Spec. & $3450-7650$ & 4.0 & 30 Jun. 2008, 06:15 & $2 \times 2400$ \\
\hline IGR J21347+4737 & 21:34:20.38 & $+47: 38: 00.2$ & Cassini+BFOSC & $3500-8700$ & 4.0 & 11 May 2008, 01:01 & $2 \times 1800$ \\
\hline
\end{tabular}

$\dagger$ Coordinates extracted from the USNO catalogues, having an accuracy of about 0!2 (Deutsch 1999; Assafin et al. 2001; Monet et al. 2003).

* Coordinates extracted from the DSS-II-Red frames, having an accuracy of $\sim 1$.

Further spectra were retrieved from two different astronomical archives: the Sloan Digitized Sky Survey ${ }^{4}$ (SDSS, AdelmanMcCarthy et al. 2007) archive, and the Six-degree Field Galaxy Survey $^{5}$ (6dFGS) archive (Jones et al. 2004). As the 6dFGS archive provides spectra which are not calibrated in flux, we used the optical photometric information in Jones et al. (2005) to calibrate the 6dFGS data presented here.

We report in Table 1 the detailed log of observations. We list in Col. 1 the name of the observed INTEGRAL sources. In Cols. 2 and 3 we give the object coordinates, extracted from the 2MASS catalogue (with an accuracy of $\leq 0$ '. 1 , according to Skrutskie et al. 2006), from the USNO catalogues (with uncertainties of about 0!'2: Deutsch 1999; Assafin et al. 2001; Monet et al. 2003), or from the DSS-II-Red astrometry (which has a precision of $\sim 1^{\prime \prime}$ ). In Col. 4 we report the telescope and the instrument used for the observations. The characteristics of each spectrograph are presented in Cols. 5 and 6. Column 7 provides the observation date and the UT time at mid-exposure, while Col. 8 reports the exposure times and the number of observations of each source.

As a complement to the information on the putative counterpart of IGR J18173-2509, we analyzed an optical $R$-band frame acquired with the ESO $3.6 \mathrm{~m}$ telescope plus EFOSC2 on 21 June 2007 (start time: 07:28 UT; duration: 20 s) under a seeing of 1 .' 0 ; the $2 \times 2$-rebinned CCD of EFOSC2 secured a plate scale of $0.31 /$ pix, and a useful field of $5.2 \times 5.2$.

This imaging frame was corrected for bias and flat-field in the usual fashion and was calibrated using nearby USNO-A2. $0^{6}$ stars. Simple aperture photometry, within the MIDAS $^{7}$ package,

\footnotetext{
${ }^{4}$ http://www.sdss.org/

5 http://www.aao.gov.au/local/www/6df/

${ }^{6}$ Available at http://archive.eso.org/skycat/servers/ usnoa/

7 http://www.eso.org/projects/esomidas
}

was then used to measure the $R$-band magnitude of the putative optical counterpart of IGR J18173-2509.

\section{Results}

Here we present the results of our spectroscopic campaign described in Sect. 2. The optical magnitudes quoted below, if not otherwise stated, are extracted from the USNO-A2.0 catalogue.

As done in Papers I-VI, we describe here the identification and classification criteria for the optical spectra of the sources considered in this work.

In order to infer the reddening along the line of sight of Galactic sources, when possible, we considered an intrinsic $\mathrm{H}_{\alpha} / \mathrm{H}_{\beta}$ line ratio of 2.86 (Osterbrock 1989) and we determined the corresponding colour excess, using the Galactic extinction law of Cardelli et al. (1989), from the comparison between the intrinsic line ratio and the measured one.

To derive the distance of the compact Galactic X-ray sources of our sample, in the case of CVs we assumed an absolute magnitude $M_{V} \sim 9$ and an intrinsic color index $(V-R)_{0} \sim 0$ mag (Warner 1995), whereas for HMXBs, when applicable, we used the intrinsic stellar color indices and absolute magnitudes as reported in Lang (1992) and Wegner (1994). For the single low-mass X-ray binary (LMXB) of our sample, we considered $(V-R)_{0} \sim 0 \sim M_{R}$ (e.g., van Paradijs \& McClintock 1995).

For the emission-line AGN classification, we used the criteria of Veilleux \& Osterbrock (1987) and the line ratio diagnostics of Ho et al. (1993, 1997) and of Kauffmann et al. (2003); moreover, for the subclass assignation of Seyfert 1 nuclei, we used the $\mathrm{H}_{\beta} /[\mathrm{O}$ III $] \lambda 5007$ line flux ratio criterion described in Winkler (1992).

The spectra of the galaxies shown here were not corrected for starlight contamination (see, e.g., Ho et al. 1993, 1997) given the limited $\mathrm{S} / \mathrm{N}$ and the spectral resolution. We do not consider that this will affect any of our main results and conclusions. 
In the following we consider a cosmology with $H_{0}=$ $65 \mathrm{~km} \mathrm{~s}^{-1} \mathrm{Mpc}^{-1}, \Omega_{\Lambda}=0.7$ and $\Omega_{\mathrm{m}}=0.3$; the luminosity distances of the extragalactic objects reported in this paper are computed with these parameters using the Cosmology Calculator of Wright (2006). Moreover, when not explicitly stated otherwise, for our X-ray flux estimates we will assume a Crab-like spectrum except for the XMM-Newton sources, for which we considered the $0.2-12 \mathrm{keV}$ flux reported in Saxton et al. (2008) or Watson et al. (2008). We also remark that the results presented here supersede the preliminary ones reported in Masetti et al. (2008c) and in Parisi et al. (2008a,b,c,d).

As in Papers IV-VI, the next subsections will deal with the object identifications by dividing them into three broad classes (CVs, X-ray Binaries and AGNs) ordered according to their increasing distance from Earth.

\section{1. $C V s$}

We identify 4 objects of our sample (IGR J08390-4833, IGR J18173-2509, IGR J18308-1232 and IGR J19267+1325) as dwarf nova CVs because of the characteristics of their optical spectra (Fig. 3). All of them show Balmer emission lines up to at least $\mathrm{H}_{\gamma}$, as well as $\mathrm{He}$ I and He II lines in emission. All of the detected lines are consistent with being at $z=0$, indicating that these objects lie within our Galaxy. In addition, source IGR J11098-6457 is identified as a symbiotic star given its optical spectral continuum, which shows the typical features of a red giant star with superimposed $\mathrm{H}_{\alpha}$ and $\mathrm{H}_{\beta}$ emissions, again at $z=0$ (Fig. 3, lower left panel). Our $R$-band photometry of the counterpart of IGR J18173-2509, described in Sect. 2, yields a magnitude $R=17.2 \pm 0.1$.

The main spectral features of these objects, and the main astrophysical parameters which can be inferred from the available optical and X-ray observational data, are given in Table 2. The X-ray luminosities listed in this Table for the various objects were computed using the fluxes reported in Voges et al. (1999), White et al. (2000), Bird et al. (2007), Landi et al. (2007b, 2008c,d), Sazonov et al. (2008), Ibarra et al. (2008a,b) and Tomsick et al. (2008b).

In the spectra of the sources identified here as dwarf nova $\mathrm{CVs}$ the $\mathrm{He}$ II $\lambda 4686 / \mathrm{H}_{\beta}$ equivalent width $(E W)$ ratio is $\gtrsim 0.5$ and the EWs of $\mathrm{He}$ II and $\mathrm{H}_{\beta}$ are around (or larger than) $10 \AA$ : this indicates that these sources are quite likely magnetic CVs belonging to the intermediate polar (IP) subclass (see Warner 1995, and references therein). Indeed, for IGR J08390-4833 and IGR J19267+1325, Sazonov et al. (2008) and Evans et al. (2008), respectively, confirmed this hypothesis by measuring the likely spin period of the white dwarf (WD) harboured in these systems.

Our findings are consistent with the results reported in Revnivtsev et al. (2008) and Steeghs et al. (2008), thus confirming their identification of IGR J08390-4833 and IGR $\mathrm{J} 19267+1325$, respectively, as magnetic dwarf nova CVs. For the latter object, however, we measure an $\mathrm{H}_{\alpha} E W$ which is a factor of $\sim 2$ lower than the value reported by Steeghs et al. (2008), obtained from a spectrum acquired about one year before ours; this long-term emission line variability is however not unusual for IP CVs (e.g., Warner 1995).

For the case of IGR J11098-6457, using the BruzualPersson-Gunn-Stryker ${ }^{8}$ (Gunn \& Stryker 1983) and JacobyHunter-Christian ${ }^{9}$ (Jacoby et al. 1984) spectroscopy atlases, we constrain the spectral type of its optical counterpart to be M2 III.

\footnotetext{
8 Available at: ftp://ftp.stsci.edu/cdbs/grid/bpgs/

9 Available at: ftp://ftp.stsci.edu/cdbs/grid/jacobi/
}

We also note a possible excess on the blue side of the optical continuum, which is a common feature in symbiotic stars.

From this spectral information, assuming colours and absolute $V$ magnitude of a M2 III star (Ducati et al. 2001; Lang 1992) and considering the measured Balmer line ratio, we obtain a distance of $\sim 20 \mathrm{kpc}$ for the source, which would place it well beyond the Carina arm of the Galaxy. This suggests that more absorption should occur along the line of sight and that this number should rather be used as an (admittedly loose) upper limit for the distance to this object. Indeed, if one assumes the total Galactic colour excess along the source line of sight, $E(B-V)_{\mathrm{Gal}}=1.042 \mathrm{mag}$ (Schlegel et al. 1998), we obtian a distance of $\sim 5.2 \mathrm{kpc}$ to the source, which is well within the aforementioned Galactic arm.

We add that, as reported in Landi et al. (2008d), a further soft X-ray source is detected with Swift/XRT in the field of IGR J11098-6457, although formally outside of the IBIS error circle. While further investigation is needed to understand whether this is the actual soft X-ray counterpart of the INTEGRAL object, the Swift/XRT observation of Landi et al. (2008d) nevertheless indicates that X-ray activity up to $10 \mathrm{keV}$ is produced by the symbiotic star identified here.

\subsection{X-ray binaries}

Four of the INTEGRAL sources selected here (IGR J11435-6109, 1ES 1210-646, IGR J17404-3655 and IGR $\mathrm{J} 21347+4737$ ) can be classified as Galactic X-ray binaries by their overall spectral appearance (see Fig. 4), which is typical of this class of objects (see e.g. Papers I-VI), with narrow Balmer and/or helium emission lines at a wavelength consistent with that of the laboratory restframe, superimposed on an intrinsically blue continuum.

The spectral shape of all of these sources but IGR $\mathrm{J} 21347+4737$ appears however substantially reddened, implying the presence of interstellar dust along the line of sight. This is quite common in X-ray binaries detected with INTEGRAL (e.g., Papers IV-VI) and indicates that these objects are relatively far from Earth. The confirmation of the presence of reddening along the line of sight towards these sources comes from either the observed $\mathrm{H}_{\alpha} / \mathrm{H}_{\beta}$ line ratio (see Table 3 ) or the observed optical colours. In all cases we indeed find a reddening compatible with the Galactic one along the line of sight of the object (Schlegel et al. 1998).

Three of the sources of our sample (IGR J11435-6109, 1ES 1210-646 and IGR J21347+4737) appear to be HMXBs due to the optical spectral features readily detected, and which point to a early-type star as the optical counterpart of these hard X-ray sources. These findings confirm the results of Negueruela et al. (2007) regarding IGR J11435-6109.

A word of caution should however be spent about the classification of 1ES 1210-646: although our optical spectroscopy excludes that this system is a magnetic dwarf nova as suggested by Revnivtsev et al. (2007) on the grounds of its Swift/XRT X-ray spectrum, the detection of an iron line at $6.7 \mathrm{keV}$ reported by these authors is rarely seen in accreting compact objects (such as neutron stars and black holes) hosted in HMXBs (but see Ribó et al. 1999; Burderi et al. 2000; Paul et al. 2002; and Naik \& Paul 2003, for detections of this line in HMXBs). However, the hypothesis of a WD as the accretor also has problems. The only case (to the best of our knowledge) of an X-ray binary composed of an early type star and a white dwarf, i.e. CI Cam (see e.g. Orlandini et al. 2000; Filippova et al. 2008, and references 

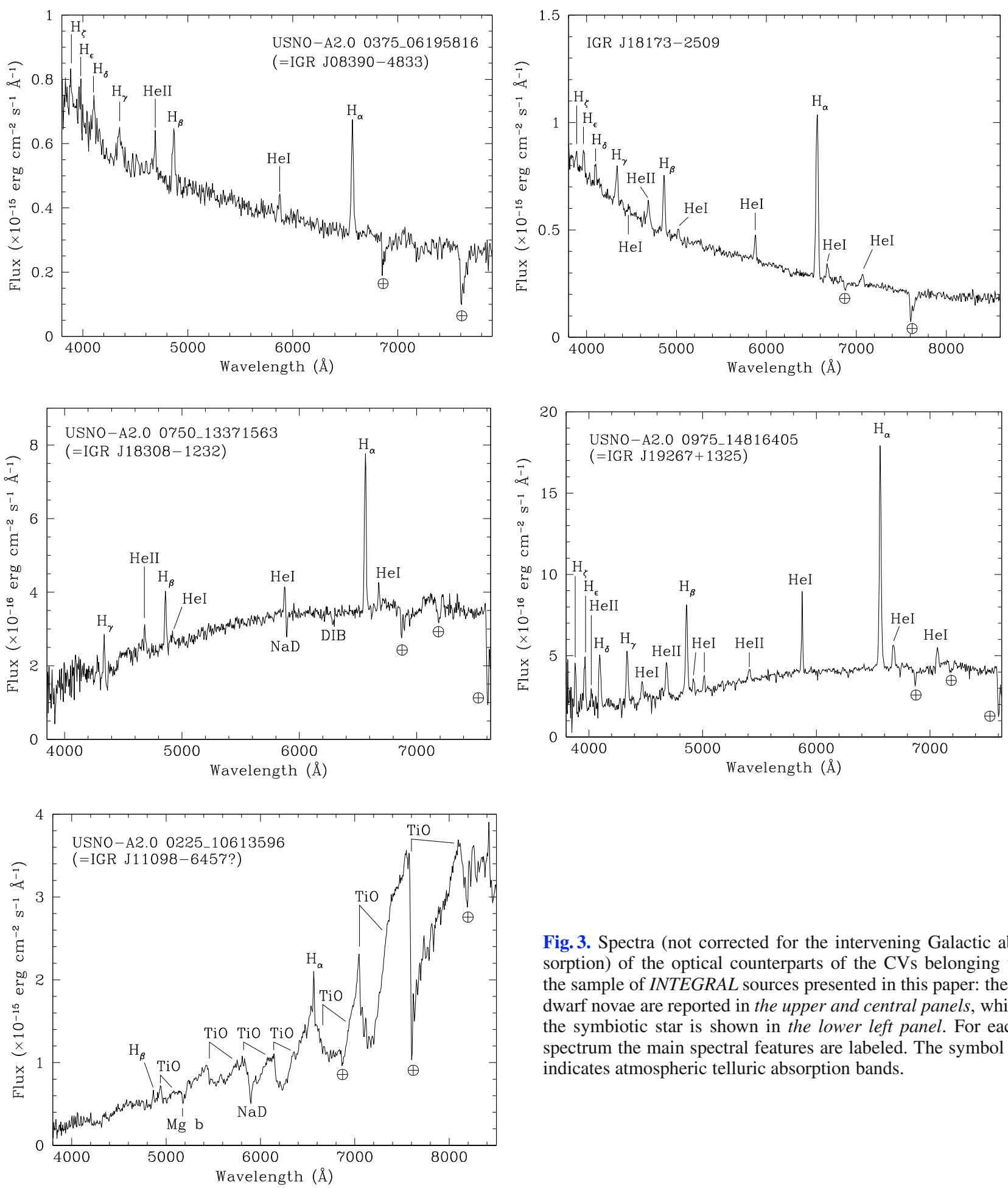

Fig. 3. Spectra (not corrected for the intervening Galactic absorption) of the optical counterparts of the CVs belonging to the sample of INTEGRAL sources presented in this paper: the 4 dwarf novae are reported in the upper and central panels, while the symbiotic star is shown in the lower left panel. For each spectrum the main spectral features are labeled. The symbol $\oplus$ indicates atmospheric telluric absorption bands.

therein) shows a completely different behaviour, both in terms of secondary star nature and persistent X-ray emission: indeed, CI Cam has a supergiant $\mathrm{B}[\mathrm{e}]$ star as the optical companion (Hynes et al. 2002); moreover, its low-level 2-10 keV luminosity (Boirin et al. 2002) is at least 2 orders of magnitude fainter than that of 1ES 1210-646 (see Table 3). Thus, we still suggest that this source is an HMXB, although with peculiarities which certainly deserve further multiwavelength studies.

We can instead rule out an HMXB nature for IGR J17404-3655 because its optical magnitudes do not fit any star of early spectral type, not even if absorption along the line of sight is considered (see Table 3). It also does not show features of a red giant star (as, for instance, in the case of IGR J11098-6457); rather, its reddened spectrum suggests that it is actually substantially absorbed by the Galactic dust, indicating that this object lies far from Earth. Besides, its emission-line spectral appearance is quite different from that of dwarf nova CVs in Fig. 3. Because of all this, we can exclude that it is a CV. We thus identify this hard X-ray source as an LMXB. 
Table 2. Synoptic table containing the main results concerning the 5 CVs (see Fig. 3) identified in the present sample of INTEGRAL sources.

\begin{tabular}{|c|c|c|c|c|c|c|c|c|c|c|}
\hline \multirow[t]{2}{*}{ Object } & \multicolumn{2}{|c|}{$\mathrm{H}_{\alpha}$} & \multicolumn{2}{|c|}{$\mathrm{H}_{\beta}$} & \multicolumn{2}{|c|}{ He II $\lambda 4686$} & \multirow{2}{*}{$\begin{array}{c}R \\
\mathrm{mag}\end{array}$} & \multirow{2}{*}{$\begin{array}{c}A_{V} \\
(\mathrm{mag})\end{array}$} & \multirow{2}{*}{$\begin{array}{c}d \\
(\mathrm{pc})\end{array}$} & \multirow[t]{2}{*}{$L_{\mathrm{X}}$} \\
\hline & $E W$ & Flux & $E W$ & Flux & $E W$ & Flux & & & & \\
\hline IGR J08390-4833 & $27 \pm 3$ & $8.6 \pm 0.8$ & $8.7 \pm 0.9$ & $4.1 \pm 0.4$ & $5.2 \pm 0.8$ & $2.6 \pm 0.4$ & 16.6 & $\sim 0$ & $\sim 330$ & $\begin{array}{r}0.46(0.5-8) \\
0.99(17-60)\end{array}$ \\
\hline IGR J11098-6457 & $5.2 \pm 0.5$ & $8.0 \pm 0.8$ & $3.6 \pm 0.7$ & $1.9 \pm 0.4$ & $<2.0$ & $<1.0$ & 15.6 & 1.23 & $<20000$ & $\begin{array}{r}<33(2-10) \\
<6200(20-100)\end{array}$ \\
\hline IGR J18173-2509 & $92 \pm 3$ & $25.0 \pm 0.8$ & $14.0 \pm 1.0$ & $6.9 \pm 0.5$ & $7.0 \pm 1.0$ & $3.6 \pm 0.5$ & 17.2 & 0.73 & $\sim 330$ & $\begin{array}{r}0.35-0.49(0.2-12) \\
1.7(2-10) \\
2.1(20-100)\end{array}$ \\
\hline IGR J18308-1232 & $21.9 \pm 1.1$ & $7.7 \pm 0.4$ & $8.5 \pm 0.9$ & $2.2 \pm 0.2$ & $3.9 \pm 0.9$ & $9.3 \pm 2.0$ & 17.0 & 0.67 & $\sim 320$ & $\begin{array}{r}0.32(0.2-12) \\
0.060(0.24-2.0) \\
1.8(20-100)\end{array}$ \\
\hline IGR J19267+1325 & $63.6 \pm 1.9$ & $26.9 \pm 0.8$ & $45 \pm 3$ & $11.8 \pm 0.8$ & $22 \pm 2$ & $5.3 \pm 0.5$ & 17.8 & $\sim 0$ & $\sim 580$ & $\begin{array}{r}0.13(0.1-2.4) \\
4.0(0.3-10) \\
3.3(2-10) \\
4.0(20-100)\end{array}$ \\
\hline
\end{tabular}

Note: $E W \mathrm{~s}$ are expressed in $\AA$, line fluxes are in units of $10^{-15} \mathrm{erg} \mathrm{cm}^{-2} \mathrm{~s}^{-1}$, whereas X-ray luminosities are in units of $10^{32}$ erg s $\mathrm{s}^{-1}$ and the reference band (between brackets) is expressed in $\mathrm{keV}$.
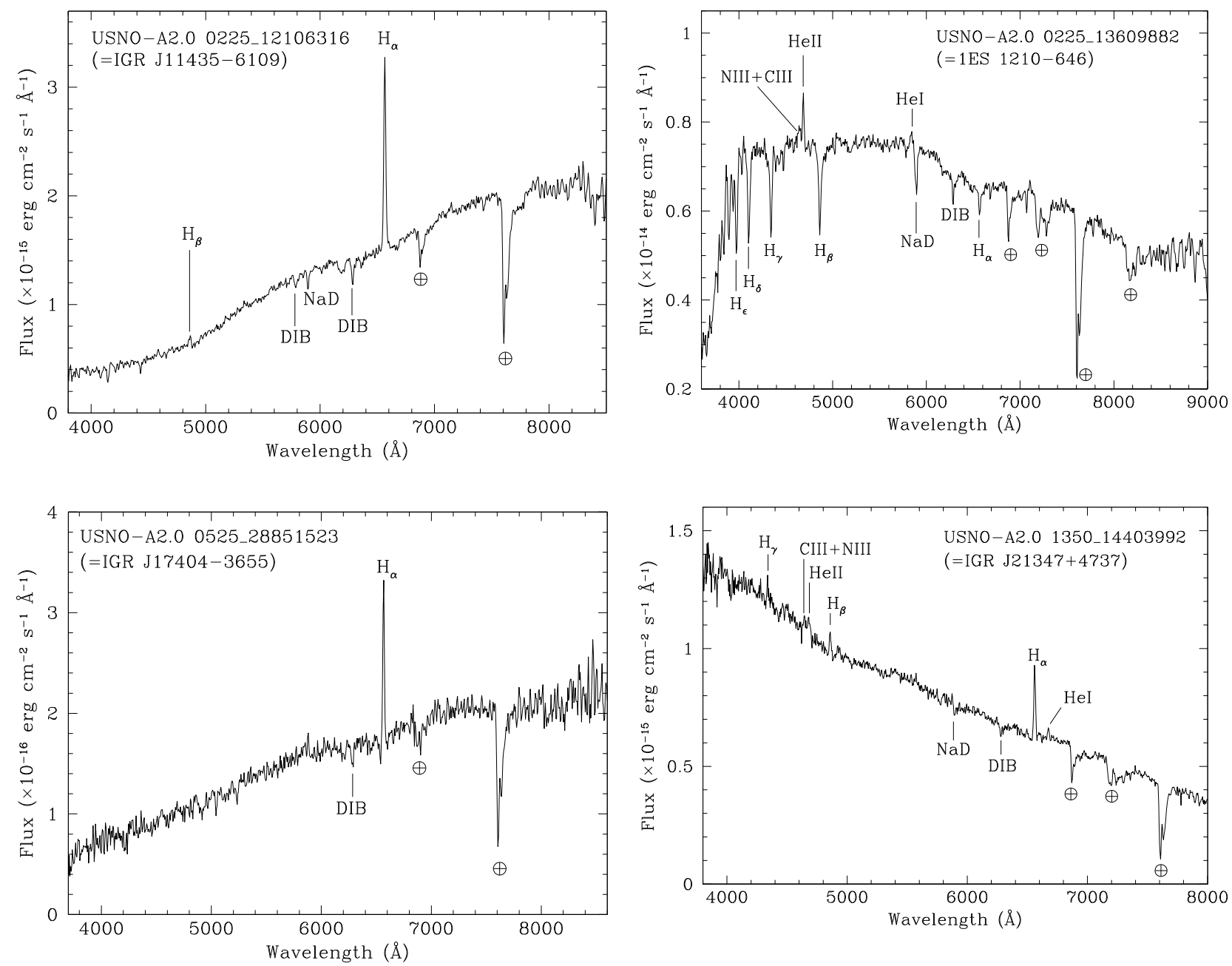

Fig. 4. Spectra (not corrected for the intervening Galactic absorption) of the optical counterparts of the X-ray binaries belonging to the sample of INTEGRAL sources presented in this paper. For each spectrum the main spectral features are labeled. The symbol $\oplus$ indicates atmospheric telluric absorption bands. 
Table 3. Synoptic table containing the main results concerning the 4 X-ray binaries (see Fig. 4) identified or observed in the present sample of INTEGRAL sources.

\begin{tabular}{|c|c|c|c|c|c|c|c|c|c|c|c|}
\hline \multirow[t]{2}{*}{ Object } & \multicolumn{2}{|c|}{$\mathrm{H}_{\alpha}$} & \multicolumn{2}{|c|}{$\overline{\mathrm{H}_{\beta}}$} & \multicolumn{2}{|c|}{ He II $\lambda 4686$} & \multirow{2}{*}{$\begin{array}{l}\text { Optical } \\
\text { mag. }\end{array}$} & \multirow{2}{*}{$\begin{array}{c}A_{V} \\
(\mathrm{mag})\end{array}$} & \multirow{2}{*}{$\begin{array}{c}d \\
(\mathrm{kpc})\end{array}$} & \multirow{2}{*}{$\begin{array}{l}\text { Spectral } \\
\text { type }\end{array}$} & \multirow[t]{2}{*}{$L_{\mathrm{X}}$} \\
\hline & $E W$ & Flux & $E W$ & $\begin{array}{l}\text { Flux } \\
\end{array}$ & $E W$ & Flux & & & & & \\
\hline IGR J11435-6109 & $25.8 \pm 1.3$ & $39 \pm 2$ & $3.5 \pm 1.1$ & $2.2 \pm 0.7$ & $<2.2$ & $<1.2$ & $16.43^{\mathrm{a}}(V)$ & 5.7 & $\sim 8.6$ & $\begin{array}{l}\text { B2 III } \\
\text { or B0 V }\end{array}$ & $\begin{array}{r}2.5(0.16-3.5) \\
8.2(0.3-10) \\
16(20-100)\end{array}$ \\
\hline 1ES 1210-646 & in abs. & in abs. & in abs. & in abs. & $2.8 \pm 0.3$ & $21 \pm 2$ & $13.9(R)$ & 3.3 & $\sim 2.8$ & B5 V & $\begin{array}{r}1.9(0.16-3.5) \\
64(1-8) \\
12(2-10) \\
1.0(20-100)\end{array}$ \\
\hline IGR J17404-3655 & $14.2 \pm 0.14$ & $2.5 \pm 0.3$ & $<9$ & $<0.9$ & $<6$ & $<0.6$ & $17.3(R)$ & 3.1 & $\sim 9.1$ & - & $\begin{array}{r}12(2-10) \\
13(20-100)\end{array}$ \\
\hline IGR J21347+4737 & $8.1 \pm 0.6$ & $5.1 \pm 0.4$ & $1.2 \pm 0.2$ & $1.2 \pm 0.2$ & $1.6 \pm 0.4$ & $1.7 \pm 0.4$ & $14.2(R)$ & 2.2 & $\sim 5.8$ & B3 V & $\begin{array}{c}0.08(0.5-8) \\
6.0(20-100)\end{array}$ \\
\hline
\end{tabular}

Note: $E W \mathrm{~s}$ are expressed in $\AA$, line fluxes are in units of $10^{-15} \mathrm{erg} \mathrm{cm}^{-2} \mathrm{~s}^{-1}$, whereas X-ray luminosities are in units of $10^{34} \mathrm{erg} \mathrm{s}^{-1}$ and the reference band (between brackets) is expressed in $\mathrm{keV}$.

${ }^{\text {a }}$ From Negueruela et al. (2007).

Table 3 collects the relevant optical spectral information on these 4 sources, along with their main parameters inferred from the available X-ray and optical data. X-ray luminosities in Table 3 were calculated using the fluxes in Forman et al. (1978), Elvis et al. (1992), Thompson et al. (1998), Reynolds et al. (1999), Bird et al. (2007), Landi et al. (2008e), Sazonov et al. (2008) and Tomsick et al. (2008a).

For the HMXBs detected in our sample we obtained the constraints for distance, reddening, spectral type and X-ray luminosity shown in Table 3 by considering the absolute magnitudes of early-type stars and by applying the method described in Paper III for the classification of source 2RXP J130159.6-635806. Given the information on the optical magnitudes and the line-ofsight reddening (see Table 3), we can state that none of these 3 objects hosts a blue supergiant as a secondary star, as this would place them far outside of the Galaxy.

Going now into detail for some of these sources, we note that inspection of the optical spectrum of 1ES 1210-646 shows that the entire Balmer series appears in absorption. However, the presence of He I, He II and N III + C III (Bowen blend) emissions makes us confident that this object is the optical counterpart of this X-ray source. We also note that the $\mathrm{H}_{\alpha}$ absorption is not very pronounced with respect to the other Balmer absorption lines: indeed, its $E W$ is around $2.5 \AA$, which is less than half of the value expected from a B5 V star (Jaschek \& Jaschek 1987). This may hint at the presence of a hidden $\mathrm{H}_{\alpha}$ emission which partially fills the corresponding absorption line. The dereddened optical spectral continuum of 1ES 1210-646 is nevertheless consistent with that of an intermediate-type, main-sequence B star.

Concerning the counterpart of IGR J21347+4737, we detect a number of emission lines in its optical spectrum: while this is the confirmation that this object is the optical counterpart of the aforementioned INTEGRAL source, the optical spectral appearance is completely different from the findings of Bikmaev et al. (2008a), who observed this source about one year earlier than we did. This suggests that the circumstellar disk around the optical companion formed again during this time lapse. This behaviour is not surprising, given the marked X-ray variability of this HMXB (Sazonov et al. 2008; Bikmaev et al. 2008a). Due to the large variability of the Balmer lines, for this source we used its USNO-A2.0 $B$ and $R$ magnitudes to determine its reddening, as we do not expect them to vary substantially with time given the HMXB nature of this source.

It is moreover noted that IGR J11435-6109 has a hydrogen column density $N_{\mathrm{H}}$, inferred by Tomsick et al. (2008a) from the source X-ray spectrum, which is $\sim 15$ times higher than the one derived from the optical reddening using the empirical formula of Predehl \& Schmitt (1995). This is often observed in absorbed HMXBs detected with INTEGRAL (e.g., Chaty 2008) and suggests the presence of additional absorbing material in the vicinity of the X-ray source, likely due to the accretion stream flowing onto the compact object in this X-ray system. Regarding this source, we also point out that both of our possible optical spectral classifications for the companion star fit with the position occupied by this object in the $\mathrm{Be} / \mathrm{X}$ locus of the Corbet diagram (Corbet 1986) on the basis of its spin and orbital periods (Swank \& Markwardt 2004; Corbet \& Remillard 2005).

None of these systems is associated with a radio source. This means that none of them is likely to be a jet-emitting X-ray binary (i.e., a microquasar).

\subsection{AGNs}

It is found that 11 objects of our sample show optical spectra that allow us to classify them as AGNs (see Table 4). Nine of these objects present strong, redshifted broad and/or narrow emission lines typical of nuclear galactic activity: seven of them can be classified as type 1 (broad-line) and two as type 2 (narrow-line) AGNs. Among type 1 AGNs, we find three Seyfert 1.5 galaxies, one Sefert 1.2 galaxy, one narrow-line (NL) Seyfert 1 galaxy and one high-redshift type 1 QSO; for the case of IGR J18249-3243, only a general Seyfert 1 classification can be given due to the contamination of its spectrum by an interloping Galactic star (see Fig. 5, lower left panel): this is similar to other cases that we previously found (Papers I and V). We moreover revise here to Seyfert 1.5 the AGN class of galaxy LEDA 166252, the optical counterpart of IGR J12415-5750. We can therefore exclude the Seyfert 2 classification reported in the literature and in on-line 
Table 4. Synoptic table containing the main results concerning the 11 AGNs (Figs. 5-7) identified or observed in the present sample of INTEGRAL sources.

\begin{tabular}{|c|c|c|c|c|c|c|c|c|}
\hline Object & $\overline{F_{\mathrm{H}_{\alpha}}}$ & $F_{\mathrm{H}_{\beta}}$ & $F_{[\mathrm{OIII}]}$ & Class & $z$ & $\overline{D_{L}(\mathrm{Mpc})}$ & $\overline{E E(B-V)_{\mathrm{Gal}}}$ & $L_{\mathrm{X}}$ \\
\hline IGR J00333+6122 & $\begin{array}{l}* \\
*\end{array}$ & $\begin{array}{l}2.5 \pm 0.6 \\
{[90 \pm 20]}\end{array}$ & $\begin{array}{c}1.76 \pm 0.09 \\
{[58 \pm 3]}\end{array}$ & Sy1.5 & 0.105 & 522.2 & 1.231 & $\begin{array}{r}22(2-10) \\
39(20-100)\end{array}$ \\
\hline Swift J0216.3+5128 & - & - & $\begin{array}{c}0.24 \pm 0.03 \\
{[0.36 \pm 0.05]}\end{array}$ & likely Sy2 & 0.422 & 2492.1 & 0.187 & $\begin{array}{r}890(0.2-12) \\
970(2-10) \\
1100(20-100)\end{array}$ \\
\hline IGR J02466-4222 & $\begin{array}{l}\text { in abs. } \\
\text { [in abs.] }\end{array}$ & $\begin{array}{l}\text { in abs. } \\
\text { [in abs.] }\end{array}$ & $\begin{array}{l}<5.0 \\
{[<5.6]}\end{array}$ & XBONG & 0.0695 & 337.4 & 0.018 & $\begin{array}{r}\sim 0.1(0.5-8) \\
\quad 42(17-60)\end{array}$ \\
\hline IGR J02524-0829 & $\begin{array}{c}2.6 \pm 0.3 \\
{[2.8 \pm 0.3]}\end{array}$ & $\begin{array}{l}\text { in abs. } \\
\text { [in abs.] }\end{array}$ & $\begin{array}{c}2.4 \pm 0.2 \\
{[2.7 \pm 0.3]}\end{array}$ & Sy2 & 0.0168 & 78.5 & 0.054 & $\begin{array}{l}0.35(2-10) \\
2.3(17-60)\end{array}$ \\
\hline IGR J05270-6631 & - & - & - & $\begin{array}{l}\text { Type } 1 \\
\text { QSO }\end{array}$ & 0.978 & 6922.4 & 0.075 & $\begin{array}{r}280(0.1-2) \\
11(2-10) \\
5600(20-40) \\
<6300(40-100)\end{array}$ \\
\hline IGR J09025-6814 & $\begin{array}{l}\text { in abs. } \\
\text { [in abs.] }\end{array}$ & $\begin{array}{l}\text { in abs. } \\
\text { [in abs.] }\end{array}$ & $\begin{array}{l}<2.6 \\
{[<3.8]}\end{array}$ & XBONG & 0.014 & 63.4 & 0.101 & $\begin{array}{r}0.0072(2-10) \\
1.2(20-100)\end{array}$ \\
\hline IGR J09253+6929 & * & $\begin{array}{c}23 \pm 5 \\
{[55 \pm 11]}\end{array}$ & $\begin{array}{c}13.2 \pm 0.7 \\
{[31.6 \pm 1.6]}\end{array}$ & Sy1.5 & 0.039 & 185.2 & 0.290 & $\begin{array}{r}0.25(2-10) \\
4.3(20-40) \\
<4.3(40-100)\end{array}$ \\
\hline IGR J10147-6354 & * & $\begin{array}{c}37 \pm 2 \\
{[81 \pm 4]}\end{array}$ & $\begin{array}{c}9.8 \pm 0.5 \\
{[21.8 \pm 1.1]}\end{array}$ & Sy1.2 & 0.202 & 1067.2 & 0.314 & $\begin{array}{r}29(2-10) \\
<31(20-40) \\
160(40-100)\end{array}$ \\
\hline IGR J12415-5750 & * & $\begin{array}{c}42 \pm 7 \\
{[300 \pm 70]}\end{array}$ & $\begin{array}{c}88 \pm 3 \\
{[588 \pm 18]}\end{array}$ & Sy 1.5 & 0.024 & 113.7 & 0.609 & $\begin{array}{r}0.52(0.1-2.4) \\
2.3(0.2-12) \\
3.5(20-100)\end{array}$ \\
\hline IGR J16426+6536 & - & $\begin{array}{l}1.89 \pm 0.09 \\
{[2.0 \pm 0.1]}\end{array}$ & $\begin{array}{c}0.78 \pm 0.04 \\
{[0.81 \pm 0.04]}\end{array}$ & NLSy1 & 0.323 & 1821.3 & 0.021 & $\begin{array}{r}44(0.2-12) \\
910(20-40) \\
<790(40-100)\end{array}$ \\
\hline IGR J18249-3243 & * & $\begin{array}{c}<0.9 \\
{[<1.5]}\end{array}$ & $\begin{array}{c}9.6 \pm 0.9 \\
{[14.5 \pm 1.5]}\end{array}$ & Sy1 & 0.355 & 2033.2 & 0.233 & $\begin{array}{r}38(0.1-2.4) \\
260(2-10) \\
390(20-100)\end{array}$ \\
\hline
\end{tabular}

Note: emission line fluxes are reported both as observed and (between square brackets) corrected for the intervening Galactic absorption $E(B-V)_{\text {Gal }}$ along the object line of sight (from Schlegel et al. 1998). Line fluxes are in units of $10^{-15} \mathrm{erg} \mathrm{cm}^{-2} \mathrm{~s}^{-1}$, whereas X-ray luminosities are in units of $10^{43} \mathrm{erg} \mathrm{s}^{-1}$ and the reference band (between round brackets) is expressed in $\mathrm{keV}$. The typical error on the redshift measurement is \pm 0.001 but for the SDSS and 6dFGS spectra, for which an uncertainty of \pm 0.0003 can be assumed.

${ }^{*}$ Heavily blended with [N II] lines.

catalogues such as $\mathrm{SIMBAD}^{10}$ and $\mathrm{NED}^{11}$. This classification also confirms the X-ray spectral description given by Malizia et al. (2007) and Winter et al. (2008) for this source and deemed too peculiar for a Seyfert 2 AGN according to these authors.

For the remaining two objects, the nuclear activity was detected only thanks to soft X-ray emission detected with Chandra or Swift, given that no or unremarkable emission lines were apparent in their optical spectra: we therefore classify them as $\mathrm{X}$-ray bright, optically normal galaxies (XBONGs, see Comastri et al. 2002).

The main observed and inferred parameters for each of these objects are reported in Table 4. In this table, X-ray luminosities were computed from the fluxes reported in Voges et al. (1999), ROSAT Team (2000), Bird et al. (2007), Krivonos et al. (2007),

\footnotetext{
${ }_{10}$ Available at http://simbad.u-strasbg.fr

11 Available at http://nedwww.ipac. caltech.edu
}

Landi et al. (2007a,b, 2008b,d), Ibarra et al. (2008a), Malizia et al. (2007), Rodriguez et al. (2008), Sazonov et al. (2008) and Watson et al. (2008). We also used the $2-10 \mathrm{keV}$ fluxes of IGR J02524-0829 and IGR J09025-6814 which are $4.8 \times 10^{-12}$ and $1.5 \times 10^{-13} \mathrm{erg} \mathrm{cm}^{-2} \mathrm{~s}^{-1}$, repsectively, according to Landi et al. (in preparation).

For 7 out of 11 reported AGNs, the redshift value was determined in this work for the first time. The redshifts of the remaining 4 sources are consistent with those reported in the literature, e.g., in the Hyperleda archive (Prugniel 2005).

Going into detail for selected sources, we see that, according to Jonker \& Kuiper (2007), two soft X-ray emitters were found with Chandra within the IBIS error circle of IGR J00333+6122; however (see also Parisi et al. 2008a), we discard source 2 of Jonker \& Kuiper (2007) as the possible counterpart of this INTEGRAL source given its relative faintness, its softness and 
N. Masetti et al.: The nature of 20 Galactic and extragalactic IGR sources. VII
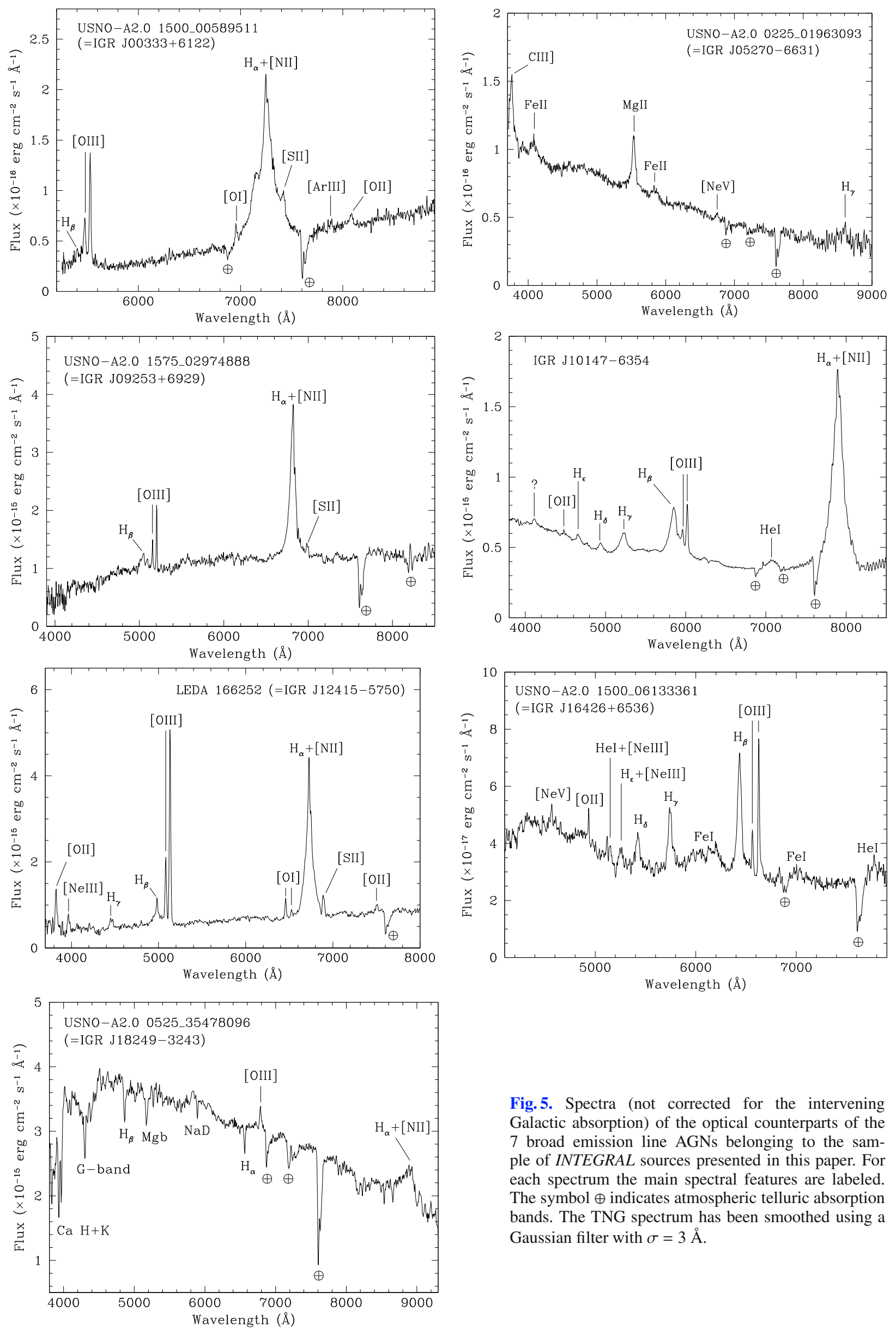

Fig. 5. Spectra (not corrected for the intervening Galactic absorption) of the optical counterparts of the 7 broad emission line AGNs belonging to the sample of INTEGRAL sources presented in this paper. For each spectrum the main spectral features are labeled. The symbol $\oplus$ indicates atmospheric telluric absorption bands. The TNG spectrum has been smoothed using a Gaussian filter with $\sigma=3 \AA$. 

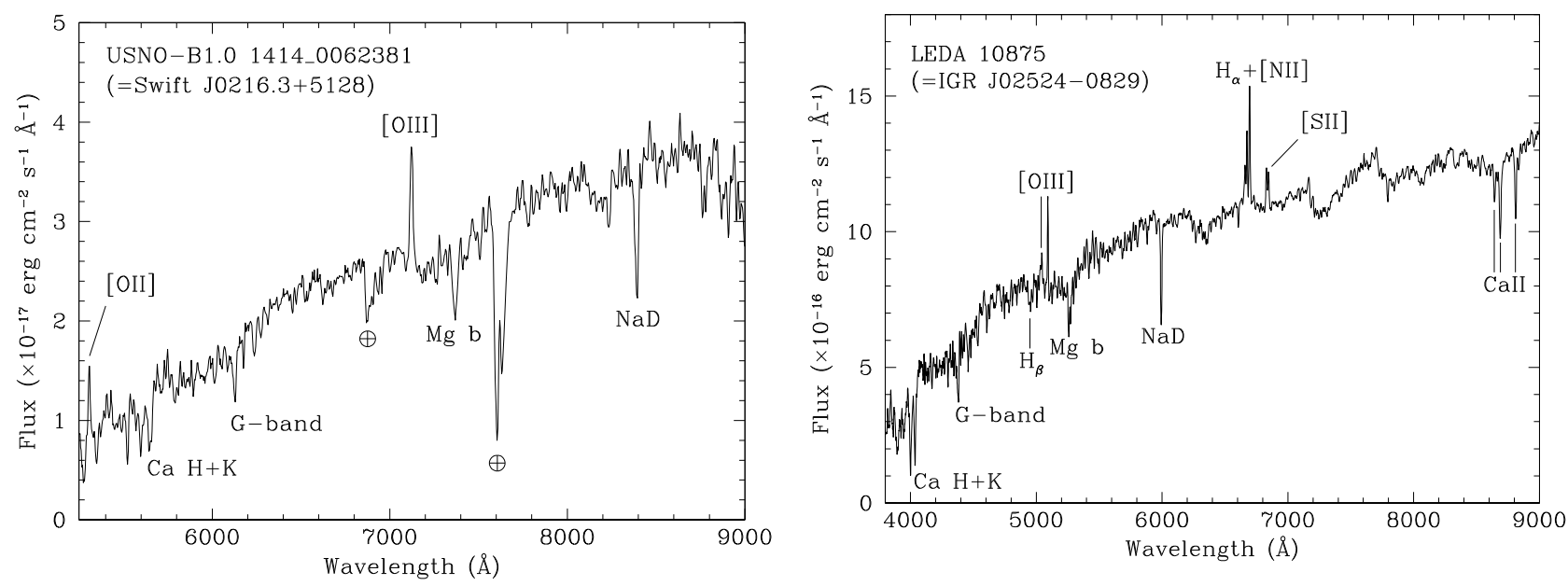

Fig. 6. Spectra (not corrected for the intervening Galactic absorption) of the optical counterparts of the 2 narrow emission line AGNs belonging to the sample of INTEGRAL sources presented in this paper. For each spectrum the main spectral features are labeled. The symbol $\oplus$ indicates atmospheric telluric absorption bands. The WHT spectrum has been smoothed using a Gaussian filter with $\sigma=3 \AA$.
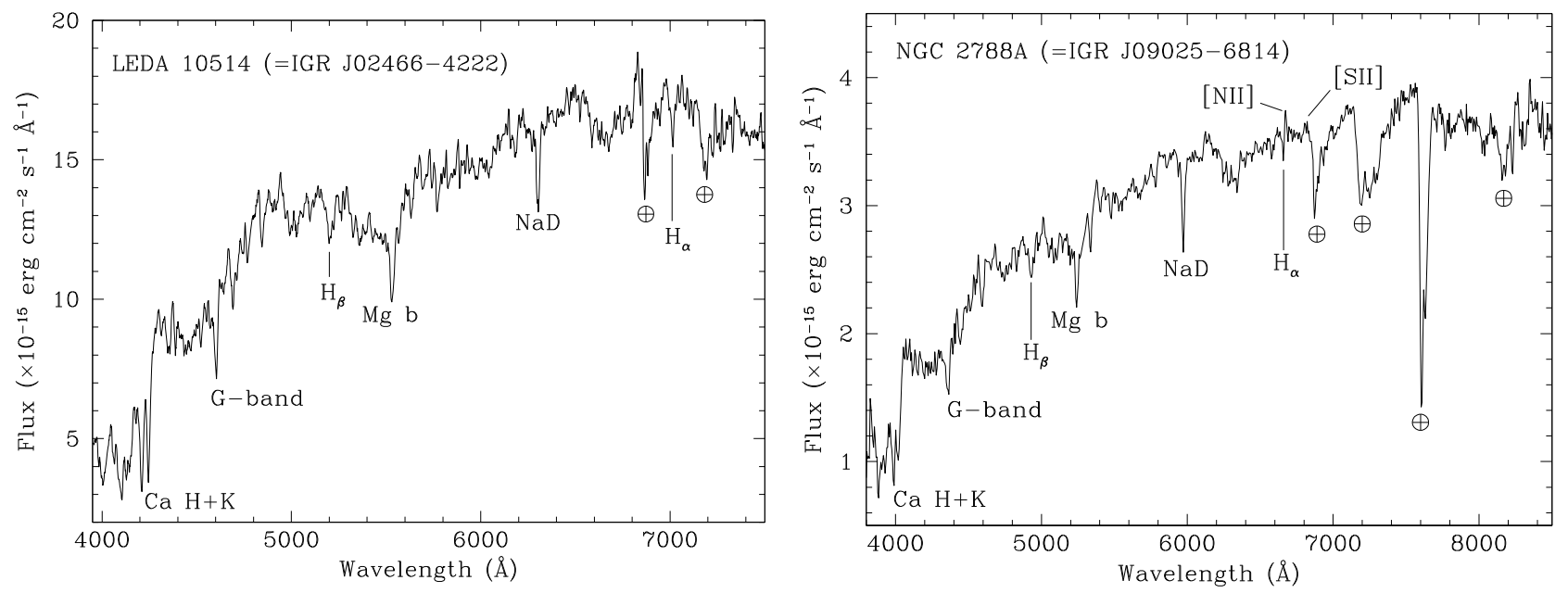

Fig. 7. Spectra (not corrected for the intervening Galactic absorption) of the optical counterparts of the 2 XBONGs belonging to the sample of INTEGRAL sources presented in this paper. For each spectrum the main spectral features are labeled. The symbol $\oplus$ indicates atmospheric telluric absorption bands.

its association with a Galactic star showing no peculiarities in its optical spectrum.

We notice here that the optical position of Swift $\mathrm{J} 0216.3+5128$ is consistent with the soft X-ray localization of Malizia et al. (2007) but not with that of Winter et al. (2008), both obtained with the same Swift/XRT observations. We checked these XRT pointings again and we found only one source within the INTEGRAL error circle of Swift $\mathrm{J} 0216.3+5128$, again at the coordinates given by Malizia et al. (2007). Moreover, given that the XMM-Newton position of Watson et al. (2008) is fully consistent with that reported by Malizia et al. (2007), we are confident that this is indeed the true localization of this source, and that we identified its correct optical counterpart.

Looking at the optical spectrum of the counterpart of Swift J0216.3+5128 we see that, at the redshift of this source, the $\mathrm{H}_{\beta}$ emission falls right in the $\mathrm{O}_{2}$ telluric band at $6870 \AA$; so, no reliable measurements of this line are possible. Nevertheless, the overall optical spectral appearance and the lack of detection of wide $\mathrm{H}_{\beta}$ emission wings allow us to classify this source as a likely Seyfert 2 AGN.
Here we confirm the identification of IGR J02524-0829 as a Seyfert 2 AGN hosted in the galaxy LEDA 10875 (Bikmaev et al. 2008b). This is done by inspecting its optical spectrum (Fig. 6, right panel) and supported by the finding of a single Swift/XRT soft X-ray source, positionally consistent with the nucleus of this galaxy, within the IBIS error box of this INTEGRAL object.

The sources IGR J02466-4222 (see also Sazonov et al. 2008) and IGR J09025-6814 could be associated with galaxies LEDA 10514 (=MCG -07-06-018) and NGC 2788A only thanks to the Chandra and Swift positions of their soft X-ray counterparts. Indeed, as it can be seen in Fig. 7, both galaxies show no peculiarities in their optical spectra: following the approach of Laurent-Muehleisen et al. (1998), we can classify both of them as "normal" galaxies. Thus, as noted above, we can state that these hard X-ray sources are XBONGs similar to NGC 4992 (=IGR J13091+1137; Paper IV). The association of NGC 2788A with a far-infrared IRAS source (IRAS 1988) further suggests this classification.

As already mentioned, we classify IGR J16426+6536 as an NL Seyfert 1 AGN, because its optical spectrum complies 
with the criteria of Osterbrock \& Pogge (1985) concerning the presence of the Fe II bump and the narrowness of the Full Width at Half Maximum (FWHM) of the $\mathrm{H}_{\beta}$ emission ( 2000 $\mathrm{km} \mathrm{s}^{-1}$ ).

It is noted that the optical counterpart of source IGR J18249-3243 lies $\sim 15$ arcsec outside the nominal ROSAT error circle. However, the Swift/XRT pointing (Landi et al. 2008b) shows that its actual soft X-ray position is definitely consistent with the proposed optical counterpart.

Unfortunately, due to the impossibility of a simultaneous detection of $\mathrm{H}_{\alpha}$ and $\mathrm{H}_{\beta}$ emission lines in the Seyfert 2 galaxies and the XBONGs of our sample, we are not able to provide an estimate of the absorption local to their AGN and, in turn, a reliable assessment of the Compton nature of these galactic nuclei with the method of Bassani et al. (1999). Thus, to explore this issue we can apply the soft-to-hard X-ray flux ratio diagnostic of Malizia et al. (2007). We find that this diagnostic has values $0.89,0.0024,0.15$ and 0.006 for Swift J0216.3+5128, IGR J02466-4222, IGR J02524-0829 and IGR J09025-6814, respectively. As a cautionary note, we remark that Malizia et al. (2007) used X-ray flux measurements in the 2-10 keV and 20$100 \mathrm{keV}$ bands to compute the aforementioned ratio, while only the $(0.5-8 \mathrm{keV}) /(17-60 \mathrm{keV})$ and the $(2-10 \mathrm{keV}) /(17-60 \mathrm{keV})$ flux ratios are avaliable in the literature for IGR J02466-4222 and IGR J02524-0829, respectively (see Table 4).

Nevertheless, if one compares these figures with those of the sample of Malizia et al. (2007, their Fig. 5), one sees that the two XBONGs of our sample fall in the low-values tail of this flux ratio distribution. This, according to Malizia et al. (2007), strongly suggests that these two sources are Compton thick AGNs and confirms the indication of Sazonov et al. (2008) concerning the nature of IGR J02466-4222. The two Seyfert 2 galaxies of our sample, instead, are very likely Compton thin AGNs.

Following Wu et al. (2004) and Kaspi et al. (2000), and McLure \& Jarvis (2002) for the case of IGR J05270-6631, we can eventually provide an estimate of the mass of the central black hole in 6 of the 7 objects classified as type 1 AGN (this procedure could not be applied to IGR J18249-3243 as no broad $\mathrm{H}_{\beta}$ emission component was detected for it). The corresponding black hole masses for these 6 cases are reported in Table 5. Here we assumed a null local absorption for all type 1 AGNs.

\subsection{Statistical considerations}

As is customary in this series of papers, we now update the broad statistical approach used in Papers V and VI by including the results presented here, along with recent spectroscopic optical and NIR identifications of INTEGRAL sources as HMXBs (Chaty et al. 2008; but see Nespoli et al. 2008b; Nespoli et al. 2008a,c), LMXBs (Torres et al. 2006; Cadolle Bel et al. 2007; Nespoli et al. 2008b; Torres et al. 2008) and AGNs (Bikmaev et al. 2008a; Burenin et al. 2008; Gonçalves et al. 2008).

It is found that, of the 129 INTEGRAL sources identified up to now through optical or NIR spectroscopy, 71 (55\%) are AGNs, with the following breakdown: 33 (i.e., $45 \%$ of the AGN identifications) are Seyfert 1 galaxies, 29 (41\%) are Seyfert 2 galaxies, while the QSO, XBONG and BL Lac subclasses are populated by 3 objects (4\%) each. There are $41 \mathrm{X}$-ray binaries, that is, $32 \%$ of all optical/NIR identifications, with a large majority, i.e. about $80 \%$, of HMXBs; 16 objects (12\%) are CVs, with 12 of them classified as definitely or likely IPs (see Papers IV-V and the present work) and 4 of symbiotic star type.

A comparison with the numbers and percentages of spectroscopically identified INTEGRAL sources in the past years (Papers V-VI) illustrates an evolution with time of the
Table 5. BLR gas velocities (in $\mathrm{km} \mathrm{s}^{-1}$ ) and central black hole masses (in units of $10^{7} M_{\odot}$ ) for 6 Seyfert 1 AGNs belonging to the sample presented in this paper.

\begin{tabular}{ccc}
\hline \hline Object & $v_{\text {BLR }}$ & $M_{\text {BH }}$ \\
\hline IGR J00333+6122 & 8100 & 35 \\
IGR J05270-6631 & 3900 & 24 \\
IGR J09253+6929 & 4900 & 4.1 \\
IGR J10147-6354 & 4800 & 42 \\
IGR J12415-5750 & 6700 & 9.2 \\
IGR J16426+6536 & 1900 & 1.1 \\
\hline
\end{tabular}

population of the classes described above. First, one can see that the number of these identifications increased by a factor of 2.4 in two years; this indicates the interest of the community in this work of systematic identification of hard X-ray sources, and stresses the utility of soft X-ray satellites (especially Swift and Chandra) for the task of refining the high-energy error box of these sources. Indeed, our experience tells us that followup soft X-ray observations of the IBIS error circle of unidentified INTEGRAL objects clearly boosted our identification record (Papers V-VI and this work).

Second, in terms of percentage population of the aforementioned classes, one can see that AGNs always constituted the largest class, increasing up to containing now the absolute majority of new identifications. They are followed by the X-ray binaries group, which on the contrary became smaller: now it contains one third of all identifications; among these, the fraction of HMXBs still by far dominates over LMXBs, although with a reduction over time. A fair share of objects is made of (magnetic) $\mathrm{CVs}$, which are constantly the third largest group of INTEGRAL sources identified through optical spectroscopy.

Third, while the percentage population of the three classes shows some differences when one makes a comparison between Paper V and Paper VI, it basically did not vary between Paper VI and the present work. This may suggest that the relative sizes of these three largest groups of sources, when identified through optical and NIR spectroscopy, reached a "stability point".

One can moreover compare the latest percentages (see Fig. 8) with those of the 370 identified objects belonging to the collection of INTEGRAL sources (Bodaghee et al. 2007) detected between 2002 and 2006. In this catalogue there are 163 (44\%) AGNs, 160 (43\%) X-ray binaries (of which 82 are LMXBs and 78 are HMXBs, i.e., $51 \%$ and $49 \%$ of this class), and $23(6.5 \%) \mathrm{CVs}$, nearly all of them being of magnetic nature (IPs or Polars, see Barlow et al. 2006; and Landi et al. 2008a).

This confirms once more the effectiveness of the method of catalogue cross-correlation plus optical and/or NIR spectroscopy follow-up in revealing the nature of unidentified INTEGRAL sources. The above is true especially for AGNs and CVs, even if in Paper VI we suggested that optical spectroscopy searches may introduce a bias against the detection of (heavily absorbed) Galactic X-ray binaries. Indeed, by looking at the results of, e.g., Hannikainen et al. (2007), Chaty et al. (2008) and Nespoli et al. (2008a,c), all of the INTEGRAL objects spectroscopically identified in the NIR bands by these authors are HMXBs.

We conclude by stressing that, of the 129 optical and NIR spectroscopic identifications mentioned in this subsection, 104 were obtained or refined within the framework of our spectroscopic follow-up program (Papers I-VI, the present work, and references therein). 


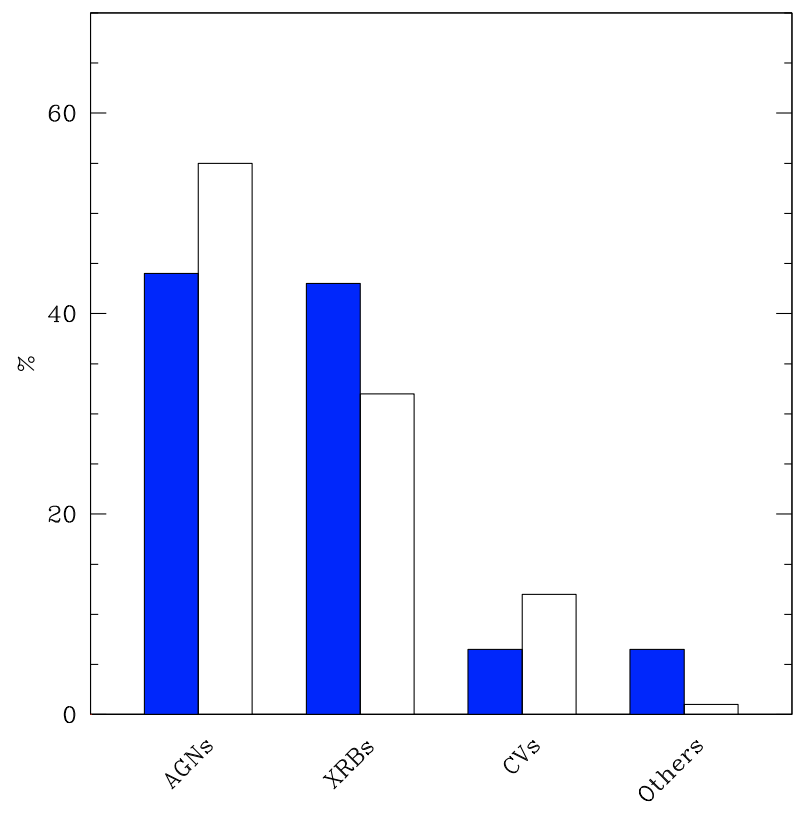

Fig. 8. Histogram, subdivided into source types, showing the percentage of INTEGRAL objects of known nature in the compilation of Bodaghee et al. (2007; left-side, darker columns), and INTEGRAL sources from various surveys and identified through optical or NIR spectroscopy (right-side, lighter columns).

\section{Conclusions}

Within our continuing identification program of INTEGRAL sources by means of optical spectroscopy (Papers I-VI) running at various telescopes around the world, in this paper we have identified and studied 20 hard X-ray objects of unknown or poorly explored nature. This was performed with the use of 6 different telescopes and using the archival data of 2 spectroscopic surveys.

We found that the selected sample is made of 11 AGNs (6 of which are Seyfert 1 galaxies, 2 are Seyfert 2 galaxies, 2 are XBONGs and one is a type 1 QSO), 4 HMXBs (all of them likely belonging to the $\mathrm{Be} / \mathrm{X}$ subclass), $4 \mathrm{CVs}$, one LMXB and one symbiotic star. Again in the present sample, as in the case of our past papers within the framework of this research, we note that the absolute majority of identified sources belongs to the AGN class, and we see a non-negligible presence of (possibly magnetic) CVs.

The results presented in this work once again demonstrate the high effectiveness of the method of catalogue crosscorrelation and/or follow-up observations with soft X-ray satellites affording arcsec-sized localizations (such as Chandra, $X M M$-Newton or Swift) plus optical (and NIR) spectroscopy to pinpoint the actual nature of the still unidentified INTEGRAL sources. Further insights can be given by deep optical or NIR Galactic surveys such as the VISTA Variables in the Via Lactea public NIR survey (VVV, Minniti et al. 2006, 2008).

Acknowledgements. We thank Silvia Galleti for Service Mode observations at the Loiano telescope; Pablo Rodríguez-Gil and Andrew Cardwell for Service Mode observations at the WHT; Francesca Ghinassi for Service Mode observations at the TNG; Antonio De Blasi and Ivan Bruni for night assistance at the Loiano telescope; Edgardo Cosgrove, Manuel Hernández and José Velásquez for day and night assistance at the CTIO telescope; Alessandro Ederoclite for support at the ESO $3.6 \mathrm{~m}$ telescope; Ariel Sánchez for night assistance at the ESO $3.6 \mathrm{~m}$ telescope; Mauro Orlandini for comments and suggestions. We also thank the anonymous referee for useful remarks which helped us to improve the quality of this paper. This research has made use of the ASI Science Data Center Multimission Archive; it also used the NASA Astrophysics
Data System Abstract Service, the NASA/IPAC Extragalactic Database (NED), and the NASA/IPAC Infrared Science Archive, which are operated by the Jet Propulsion Laboratory, California Institute of Technology, under contract with the National Aeronautics and Space Administration. This publication made use of data products from the Two Micron All Sky Survey (2MASS), which is a joint project of the University of Massachusetts and the Infrared Processing and Analysis Center/California Institute of Technology, funded by the National Aeronautics and Space Administration and the National Science Foundation. This research has also made use of data extracted from the 6dF Galaxy Survey and the Sloan Digitized Sky Survey archives; it has also made use of the ESO Science Archive operated at Garching bei München, Germany, of the SIMBAD database operated at CDS, Strasbourg, France, and of the HyperLeda catalogue operated at the Observatoire de Lyon, France. The authors acknowledge the ASI and INAF financial support via grant No. I/023/05/0. P.P. is supported by the ASI-INTEGRAL grant No. I/008/07. L.M. is supported by the University of Padua through grant No. CPDR061795/06. V.C. is supported by the CONACYT research grant 54480-F (México). D.M. is supported by the Basal CATA PFB 06/09, and FONDAP Center for Astrophysics grant No. 15010003. N.M. and L.M. thank the Pontificia Universidad Católica de Chile for the pleasant hospitality in Santiago de Chile during the preparation of this paper.

\section{References}

Adelman-McCarthy, J. K., Agüeros, M. A., Allam, S. S., et al. 2007, ApJS, 172, 634

Assafin, M., Andrei, A. H., Vieira Martins, R., et al. 2001, ApJ, 552, 380

Barlow, E. J., Knigge, C., Bird, A. J., et al. 2006, MNRAS, 372, 224

Bassani, L., Dadina, M., Maiolino, R., et al. 1999, ApJS, 121, 473

Bikmaev, I. F., Burenin, R. A., Revnivtsev, M. G., et al. 2008a, Astron. Lett., 34, 653

Bikmaev, I., Revnivtsev, M., Burenin, R., et al. 2008b, ATel, 1363

Bird, A. J., Malizia, A., Bazzano, A., et al. 2007, ApJS, 170, 175

Bodaghee, A., Courvoisier, T. J. -L., Rodriguez, J., et al. 2007, A\&A, 467, 585

Boirin, L., Parmar, A. N., Oosterbroek, T., et al. 2002, A\&A, 394, 205

Bonnet-Bidaud, J. M., de Martino, D., Falanga, M., Mouchet, M., \& Masetti, N. 2007, A\&A, 473, 185

Burderi, L., Di Salvo, T., Robba, N. R., La Barbera, A., \& Guainazzi, M. 2000, ApJ, 530, 429

Burenin, R. A., Bikmaev, I. F., Revnivtsev, M. G., et al. 2008, Astron. Lett., in press [arXiv:0810.2512]

Cadolle Bel, M., Ribó, M., Rodriguez, J., et al. 2007, ApJ, 659, 549

Cardelli, J. A., Clayton, G. C., \& Mathis, J. S. 1989, ApJ, 345, 245

Chaty, S. 2008, ChJAA Suppl., 8, 197

Chaty, S., Rahoui, F., Foellmi, C., et al. 2008, A\&A, 484, 783

Comastri, A., Mignoli, M., Ciliegi, P., et al. 2002, ApJ, 571, 771

Corbet, R. H. D. 1986, MNRAS, 220, 1047

Corbet, R. H. D., \& Remillard, R. 2005, ATel 377

Deutsch, E. W. 1999, AJ, 118, 1882

Ducati, J. R., Bevilacqua, C. M., Rembold, S. B., \& Ribeiro, D. 2001, ApJ, 558, 309

Elvis, M., Plummer, D., Schachter, J., \& Fabbiano, G. 1992, ApJS, 80, 257

Evans, P. A., Beardmore, A. P., \& Osborne, J. P. 2008, ATel, 1669

Filippova, E. V., Revnivtsev, M. G., \& Lutovinov, A. A., 2008, Astron. Lett., 34, 797

Forman, W., Jones, C., Cominsky, L., et al. 1978, ApJS, 38, 357

Gonçalves, T. S., Martin, D. C., Halpern, J. P., Eracleous, M., \& Pavlov, G. G. 2008, ATel, 1623

Gros, A., Goldwurm, A., Cadolle-Bel, M., et al. 2003, A\&A, 411, L179

Gunn, J. E., \& Stryker, L. L. 1983, ApJS, 52, 121

Hannikainen, D. C., Rawlings, M. G., Muhli, P., et al. 2007, MNRAS, 380, 665

Ho, L. C., Filippenko, A. V., \& Sargent, W. L.W. 1993, ApJ, 417, 63

Ho, L. C., Filippenko, A. V., \& Sargent, W. L.W. 1997, ApJS, 112, 315

Horne, K. 1986, PASP, 98, 609

Hynes, R. I., Clark, J. S., Barsukova, E. A., et al. 2002, ApJ, 392, 991

Ibarra, A., Kuulkers, E., \& Saxton, R. 2008a, ATel, 1397

Ibarra, A., Kuulkers, E., \& Saxton, R. 2008b, ATel, 1527

IRAS, 1988. The Point Source Catalogue, version 2.0, NASA RP-1190

Jacoby, G. H., Hunter, D. A., \& Christian, C. A. 1984, ApJS, 56, 257

Jaschek, C., \& Jaschek, M. 1987, The Classification of Stars (Cambridge:

Cambridge Univ. Press)

Jones, D. H., Saunders, W., Colless, M., et al. 2004, MNRAS, 355, 747

Jones, D. H., Saunders, W., Read, M., \& Colless, M. 2005, PASA, 22, 277

Jonker, P. G., \& Kuiper, L. 2007, ATel, 1326

Kaspi, S., Smith, P. S., Netzer, H., et al. 2000, ApJ, 533, 631

Kauffmann, G., Heckman, T. M., Tremonti, C., et al. 2003, MNRAS, 346, 1055

Krivonos, R., Revnivtsev, M., Lutovinov, A., et al. 2007, A\&A, 475, 775 
Landi, R., Masetti, N., Sguera, V., et al. 2007a, ATel, 1322

Landi, R., Masetti, N., Sguera, V., et al. 2007b, ATel, 1323

Landi, R., Bassani, L., Dean, A. J., et al. 2008a, MNRAS, in press [arXiv:0810.1844]

Landi, R., Stephen, J. B., Masetti, N., et al. 2008b, A\&A, in press [arXiv:0811.2318]

Landi, R., Masetti, N., Malizia, A., et al. 2008c, ATel, 1437

Landi, R., Masetti, N., Malizia, A., et al. 2008d, ATel, 1538

Landi, R., Masetti, N., Malizia, A., et al. 2008e, ATel, 1539

Lang, K. R. 1992, Astrophysical Data: Planets and Stars (New York: SpringerVerlag)

Laurent-Muehleisen, S. A., Kollgaard, R. I., Ciardullo, R., et al. 1998, ApJS, 118,127

Leyder, J.-C., Walter, R., Lazos, M., Masetti, N., \& Produit, N. 2007, A\&A, 465, L35

Malizia, A., Landi, R., Bassani, L., et al. 2007, ApJ, 668, 81

Masetti, N., Palazzi, E., Bassani, L., Malizia, A., \& Stephen, J. B. 2004, A\&A, 426, L41 (Paper I)

Masetti, N., Mason, E., Bassani, L., et al. 2006a, A\&A, 448, 547 (Paper II)

Masetti, N., Pretorius, M. L., Palazzi, E., et al. 2006b, A\&A, 449, 1139 (Paper III)

Masetti, N., Bassani, L., Bazzano, A., et al. 2006c, A\&A, 455, 11 (Paper IV

Masetti, N., Morelli, L., Palazzi, E., et al. 2006d, A\&A, 459, 21 (Paper V)

Masetti, N., Landi, R., Pretorius, M. L., et al. 2007, A\&A, 470, 331

Masetti, N., Mason, E., Morelli, L., et al. 2008a, A\&A, 482, 113 (Paper VI)

Masetti, N., Mason, E., Landi, R., et al. 2008b, A\&A, 480, 715

Masetti, N., Parisi, P., Palazzi, E., et al. 2008c, ATel 1620

McLure, R. J., \& Jarvis, M. J. 2002, MNRAS, 337, 109

Minniti, D., Lucas, P., Adamson, A., et al. 2006, Mem. Soc. Astron. It., 77, 1184

Minniti, D., Lucas, P., Ahumada, A. V., et al. 2008, Rev. Mex. Astron. Astrofís., in press

Monet, D. G., Levine, S. E., Canzian, B., et al. 2003, AJ, 125, 984

Naik, S., \& Paul, B. 2003, A\&A, 401, 265

Negueruela, I., Torrejón, J. M., \& McBride, V. 2007, ATel, 1239

Nespoli, E., Fabregat, J., \& Mennickent, R. E. 2008a, ATel, 1396

Nespoli, E., Fabregat, J., \& Mennickent, R. E. 2008b, ATel, 1450

Nespoli, E., Fabregat, J., \& Mennickent, R. E. 2008c, A\&A, 486, 911

Orlandini, M., Parmar, A. N., Frontera, F., et al. 2000, A\&A, 356, 163

Osterbrock, D. E. 1989, Astrophysics of Gaseous Nebulae and Active Galactic Nuclei (Mill Valley: Univ. Science Books)

Osterbrock, D. E., \& Pogge, R. W. 1985, ApJ, 297, 166

Parisi, P., Masetti, N., Landi, R., et al. 2008a, ATel, 1375

Parisi, P., Masetti, N., Landi, R., et al. 2008b, ATel, 1540

Parisi, P., Masetti, N., Jiménez, E., et al. 2008c, ATel, 1710

Parisi, P., Masetti, N., Malizia, A., et al. 2008d, ATel, 1800

Paul, B., Nagase, F., Endo, T., et al. 2002, ApJ, 579, 411

Predehl, P., \& Schmitt, J. H. M. M. 1995, A\&A, 293, 889
Prugniel, P. 2005, The Hyperleda Catalogue,

http: //leda.univ-lyon 1 . fr

Reynolds, A. P., Parmar, A. N., Hakala, P. J., et al. 1999, A\&AS, 134, 287

Revnivtsev, M. G., Sazonov, S. Y., Gilfanov, M. R., \& Sunyaev, R. A. 2003, Astron. Lett., 29, 587

Revnivtsev, M., Sunyaev, R., Lutovinov, A., \& Sazonov, S. 2007, ATel, 1253

Revnivtsev, M., Kniazev, A., Sazonov, S., et al. 2008, Astron. Lett., in press [arXiv: 0810.1434]

Ribó, M., Reig, P., Martí, J., \& Paredes, J. M. 1999, A\&A, 347, 518

Rodriguez, J., Tomsick, J. A., \& Chaty, S. 2008, A\&A, 482, 731

ROSAT Team 2000, ROSAT News No. 72, The Second ROSAT/PSPC Source

Catalogue of Pointed Observations

Saxton R. D., Read, A. M., Esquej, P., et al. 2008, A\&A, 480, 611

Sazonov, S., Revnivtsev, M., Burenin, R., et al. 2008, A\&A, 487, 509

Schlegel, D. J., Finkbeiner, D. P., \& Davis, M. 1998, ApJ, 500, 525

Skrutskie, M. F., Cutri, R. M., Stiening, R., et al. 2006, AJ, 131, 1163

Sguera, V., Bazzano, A., Bird, A. J., et al. 2006, ApJ, 646, 452

Sidoli, L., Romano, P., Mangano, V., et al. 2008, ApJ, 687, 1230

Steeghs, D., Knigge, C., Drew, J., et al. 2008, ATel, 1653

Swank, J. H., \& Markwardt, C. B. 2004, ATel, 359

Thompson, R. J., Shelton, R. G., \& Arning, C. A. 1998, AJ, 115, 2587

Tomsick, J. A., Chaty, S., Rodriguez, J., Walter, R., \& Kaaret, P. 2008a, ApJ, 685,1143

Tomsick, J. A., Rodriguez, J., Chaty, S., Walter, R., \& Kaaret, P. 2008b, ATel, 1649

Torres, M. A. P., Steeghs, D., McClintock, J., et al. 2006, ATel, 749

Torres, M. A. P., Jonker, P. G., Steeghs, D., et al. 2008, ApJ, 672, 1079

Ubertini, P., Lebrun, F., Di Cocco, G., et al. 2003, A\&A, 411, L131

van Paradijs, J., \& McClintock, J. E. 1995, Optical and ultraviolet observations of X-ray binaries, in X-ray Binaries, ed. W. H. G. Lewin, J. van Paradijs, \& E. P. J. van den Heuvel (Cambridge: Cambridge University Press), 58

Veilleux, S., \& Osterbrock, D. E. 1987, ApJS, 63, 295

Voges, W., Aschenbach, B., Boller, T., et al. 1999, A\&A, 349, 389

Walter, R. 2007, Ap. Space Sci., 309, 5

Warner, B. 1995, Cataclysmic variable stars (Cambridge: Cambridge University Press)

Watson, M. G., Schröder, A. C., Fyfe, D., et al. 2008, A\&A, in press [arXiv: 0807.1067]

Wegner, W. 1994, MNRAS, 270, 229

White, N. E., Giommi, P., \& Angelini, L. 2000, The WGACAT version of the ROSAT PSPC Catalogue, Rev. 1

Winkler, H. 1992, MNRAS, 257, 677

Winkler, C., Courvoisier, T. J.-L., Di Cocco, G., et al. 2003, A\&A, 411, L1

Winter, L. M., Mushotzky, R. F., Tueller, J., \& Markwardt, C. 2008, ApJ, 674, 686

Wright, E. L. 2006, PASP, 118, 1711

Wu, X.-B., Wang, R., Kong, M. Z., Liu, F. K., \& Han, J. L. 2004, A\&A, 424, 793 